\title{
Effectiveness and Safety of a Novel Care Model for the Management of Type 2 Diabetes at 1 Year: An Open-Label, Non-Randomized, Controlled Study
}

\author{
Sarah J. Hallberg · Amy L. McKenzie · Paul T. Williams • \\ Nasir H. Bhanpuri · Anne L. Peters • Wayne W. Campbell • Tamara L. Hazbun • \\ Brittanie M. Volk · James P. McCarter · Stephen D. Phinney • \\ Jeff S. Volek
}

Received: December 28, 2017 / Published online: February 7, 2018

(c) The Author(s) 2018. This article is an open access publication

\begin{abstract}
Introduction: Carbohydrate restriction markedly improves glycemic control in patients with type 2 diabetes (T2D) but necessitates prompt medication changes. Therefore, we assessed the effectiveness and safety of a novel care model providing continuous remote care with medication management based on biometric feedback combined with the metabolic approach of nutritional ketosis for T2D management.
\end{abstract}

Enhanced content To view enhanced content for this article go to https://doi.org/10.6084/m9.figshare. 5803119.

Electronic supplementary material The online version of this article (https://doi.org/10.1007/s13300018-0373-9) contains supplementary material, which is available to authorized users.

S. J. Hallberg · T. L. Hazbun

Medically Supervised Weight Loss, Indiana

University Health Arnett, Lafayette, IN, USA

S. J. Hallberg · A. L. McKenzie ( $\varangle)$.

N. H. Bhanpuri · B. M. Volk - J. P. McCarter .

S. D. Phinney · J. S. Volek

Virta Health, San Francisco, CA, USA

e-mail: amy@virtahealth.com

P. T. Williams

Independent Consultant, Lafayette, CA, USA

A. L. Peters

Keck School of Medicine, University of Southern

California, Los Angeles, CA, USA
Methods: We conducted an open-label, nonrandomized, controlled, before-and-after 1-year study of this continuous care intervention (CCI) and usual care (UC). Primary outcomes were glycosylated hemoglobin $\left(\mathrm{HbA}_{1 \mathrm{c}}\right)$, weight, and medication use. Secondary outcomes included fasting serum glucose and insulin, HOMA-IR, blood lipids and lipoproteins, liver and kidney function markers, and high-sensitivity C-reactive protein (hsCRP).

Results: 349 adults with T2D enrolled: CCI: $n=262$ [mean (SD); $54 \quad(8)$ years, 116.5 (25.9) $\mathrm{kg}, 40.4(8.8) \mathrm{kg} \mathrm{m}^{2}, 92 \%$ obese, $88 \%$ prescribed T2D medication]; UC: $n=87 \quad(52$ (10) years, $105.6(22.15) \mathrm{kg}, 36.72(7.26) \mathrm{kg} \mathrm{m}^{2}$, $82 \%$ obese, $87 \%$ prescribed T2D medication]. 218 participants $(83 \%)$ remained enrolled in the CCI at 1 year. Intention-to-treat analysis of the $\mathrm{CCI}$ (mean $\pm \mathrm{SE}$ ) revealed $\mathrm{HbA}_{1 \mathrm{c}}$ declined from

W. W. Campbell

Department of Nutrition Science, Purdue

University, West Lafayette, IN, USA

J. P. McCarter

Department of Genetics, Washington University School of Medicine, St. Louis, MO, USA

J. S. Volek

Department of Human Sciences, The Ohio State University, Columbus, OH, USA 
$59.6 \pm 1.0$ to $45.2 \pm 0.8 \mathrm{mmol} \mathrm{mol}{ }^{-1} \quad(7.6 \pm$ $0.09 \%$ to $6.3 \pm 0.07 \%, P<1.0 \times 10^{-16}$ ), weight declined $13.8 \pm 0.71 \mathrm{~kg}\left(P<1.0 \times 10^{-16}\right)$, and T2D medication prescription other than metformin declined from $56.9 \pm 3.1 \%$ to $29.7 \pm$ $3.0 \%\left(P<1.0 \times 10^{-16}\right)$. Insulin therapy was reduced or eliminated in $94 \%$ of users; sulfonylureas were entirely eliminated in the CCI. No adverse events were attributed to the CCI. Additional CCI 1-year effects were HOMA-IR $-55 \%\left(P=3.2 \times 10^{-5}\right)$, hsCRP $-39 \%(P<1.0$ $\left.\times 10^{-16}\right)$, triglycerides $-24 \%\left(P<1.0 \times 10^{-16}\right)$, HDL-cholesterol $+18 \%\left(P<1.0 \times 10^{-16}\right)$, and LDL-cholesterol $+10 \%\left(P=5.1 \times 10^{-5}\right)$; serum creatinine and liver enzymes (ALT, AST, and ALP) declined $(P \leq 0.0001)$, and apolipoprotein B was unchanged $(P=0.37)$. UC participants had no significant changes in biomarkers or T2D medication prescription at 1 year.

Conclusions: These results demonstrate that a novel metabolic and continuous remote care model can support adults with T2D to safely improve $\mathrm{HbA}_{1 \mathrm{c}}$, weight, and other biomarkers while reducing diabetes medication use.

ClinicalTrials.gov Identifier: NCT02519309.

Funding: Virta Health Corp.

\section{PLAIN LANGUAGE SUMMARY}

Treatments for type 2 diabetes (T2D) have improved, yet $\mathrm{T} 2 \mathrm{D}$ and being overweight are still significant public health concerns. Blood sugar in patients with T2D can improve quickly when patients eat significantly fewer dietary carbohydrates. However, this demands careful medicine management by doctors, and patients need support and frequent contact with health providers to sustain this way of living. The purpose of this study was to evaluate if a new care model with very low dietary carbohydrate intake and continuous supervision by a health coach and doctor could safely lower HbA1c, weight and need for medicines after 1 year in adults with T2D. 262 adults with T2D volunteered to participate in this continuous care intervention (CCI) along with 87 adults with T2D receiving usual care (UC) from their doctors and diabetes education program. After 1 year, patients in the CCI, on average, lowered HbA1c from 7.6 to $6.3 \%$, lost $12 \%$ of their body weight, and reduced diabetes medicine use. $94 \%$ of patients who were prescribed insulin reduced or stopped their insulin use, and sulfonylureas were eliminated in all patients. Participants in the UC group had no changes to $\mathrm{HbA1c}$, weight or diabetes medicine use over the year. These changes in CCI participants happened safely while dyslipidemia and markers of inflammation and liver function improved. This suggests the novel care model studied here using dietary carbohydrate restriction and continuous remote care can safely support adults with T2D to lower HbA1c, weight, and medicine use.

Keywords: Beta-hydroxybutyrate; Carbohydrate restriction; HbA1c; Ketosis; Type 2 diabetes; Weight loss

\begin{tabular}{|c|c|}
\hline \multicolumn{2}{|c|}{ Abbreviations } \\
\hline ALP & Alkaline phosphatase \\
\hline ALT & Alanine aminotransferase \\
\hline ApoB & Apolipoprotein B \\
\hline AST & Aspartate aminotransferase \\
\hline BHB & Beta-hydroxybutyrate \\
\hline BUN & Blood urea nitrogen \\
\hline $\mathrm{CBC}$ & Complete blood count \\
\hline CCI & Continuous care intervention \\
\hline CCI-onsite & $\begin{array}{l}\text { Subset of participants who selected } \\
\text { on-site education }\end{array}$ \\
\hline CCI-web & $\begin{array}{l}\text { Subset of participants who selected } \\
\text { web-based education }\end{array}$ \\
\hline CMP & Complete metabolic panel \\
\hline DPP-4 & Dipeptidyl peptidase- 4 inhibitor \\
\hline eGFR & $\begin{array}{l}\text { Estimated glomerular filtration } \\
\text { rate }\end{array}$ \\
\hline FT4 & Free $\mathrm{T} 4$ \\
\hline GLP-1 & $\begin{array}{l}\text { Glucagon-like peptide } 1 \text { receptor } \\
\text { agonists }\end{array}$ \\
\hline HOMA-IR & $\begin{array}{l}\text { Homeostatic model assessment of } \\
\text { insulin resistance }\end{array}$ \\
\hline hsCRP & High-sensitivity C-reactive protein \\
\hline PCP & Primary care provider \\
\hline SGLT-2 & $\begin{array}{l}\text { Sodium glucose co-transporter } 2 \\
\text { inhibitors }\end{array}$ \\
\hline $\mathrm{T} 2 \mathrm{D}$ & Type 2 diabetes \\
\hline
\end{tabular}

Abbreviations

ALP

ALT

ApoB

AST

BHB

CCI

Complete blood count

Subset of participants who selected on-site education web-based education

CMP Complete metabolic pane

DPP-4 Dipeptidyl peptidase-4 inhibitor Type 2 diabetes 
TSH

UC

Thyroid stimulating hormone

VLCD

Usual care

Very low energy diet

\section{INTRODUCTION}

The number of people living with diabetes worldwide nearly quadrupled since 1980, estimated at 422 million in 2014 [1]. In the USA, the Centers for Disease Control reports 30.3 million adults presently live with diabetes, and it is among the leading causes of death [2]. Treatment modalities for type 2 diabetes (T2D) have demonstrated varying success. Intensive lifestyle interventions are effective treatments for obese individuals with T2D when weight loss is achieved and sustained [3]. Evidence for improved cardiovascular outcomes in patients with T2D prescribed glucagon-like peptide 1 receptor agonists (GLP-1) and sodium glucose co-transporter 2 inhibitors (SGLT-2) is increasing $[4,5]$. Forty percent of patients undergoing bariatric surgery demonstrate substantial improvements in glycemic control after 1 year and many achieve T2D remission [6]. Despite advancements in treatment options, cost, side effects, adherence, and disease progression remain barriers.

Guidelines for T2D management recommend lifestyle change and weight loss $[7,8]$. However, a fraction of individuals are successful at long-term weight loss maintenance and true disease remission is uncommon [3, 9]. Mediterranean-style, DASH, and plant-based diets, sometimes with prescribed energy restriction, are recommended, but effectiveness data are limited [7] and low fat diets have not been shown to be superior for weight loss [10]. Commercially available weight loss programs have demonstrated short-term success in glycemic control, but continued success at 1 year is uncommon [11].

Glycemic control can be achieved quickly with carbohydrate restriction via very low energy diets (400-800 kcal day ${ }^{-1}$; VLCD) [12]. However, VLCD are necessarily temporary and outcomes often revert when patients resume former dietary patterns. Alternatively, nutritional ketosis, achieved by consuming moderate protein and greatly reduced carbohydrate, results in similarly increased serum betahydroxybutyrate (BHB) concentrations as observed during VLCD, which signifies a shift to using fat as the body's primary fuel source [12]. This nutritional therapy may help patients achieve sustainable glycemic control without prescribed energy restriction. Benefit may accrue from decreased circulating glucose and insulin [13], ketone signaling [14, 15], or eventual weight loss. Studies utilizing carbohydrate restriction observed improved glycemic control and cardiometabolic markers, but were often short-term trials of small groups, excluded subjects prescribed insulin, or infrequently monitored or achieved ketosis [16-20].

The chronic nature of diabetes care presents an additional challenge requiring sustained behavioral change that is difficult to support with traditional medical care including infrequent provider contact [21]. Adherence to lifestyle changes may be poor in the absence of support from providers and peers. We therefore hypothesized that a comprehensive care model that supports patients to achieve sustained nutritional ketosis while eating to satiety may have robust benefits in T2D management. This intervention utilizes continuous care through intensive, digitally enabled support including telemedicine access to a medical provider (physician or nurse practitioner), health coaching, nutrition and behavior change education and individualized care plans, biometric feedback, and peer support via an online community. Thus, the purpose of this study was to assess the effectiveness and safety of a novel care model (Virta Clinic, Virta Health; San Francisco, CA, USA) for the management of T2D after 1 year. Secondary aims were (1) to determine if a difference in primary outcomes existed between participants who self-selected on-site versus web-based education delivery and (2) explore the time course of biomarker change at 70 days and 1 year into the CCI. Primary endpoints to assess effectiveness of the intervention were change in glycosylated hemoglobin $\left(\mathrm{HbA}_{1 \mathrm{c}}\right)$, body weight, and medication prescription after 1 year. Secondary outcomes, including clinical biomarkers of 
associated physiological systems and adverse events, were assessed to determine safety of the intervention.

\section{METHODS}

We utilized an open-label, non-randomized, controlled, before-and-after study design with a cohort of patients who self-selected to participate in the metabolic and continuous care intervention (CCI) for T2D and a comparison group of patients who self-selected to participate while receiving their usual care (UC) from their own medical providers and diabetes education program (Clinicaltrials.gov Identifier NCT02519309). Adults diagnosed with T2D were recruited via clinical referrals, local advertisements, and word of mouth in Lafayette, Indiana, USA and surrounding region from August 2015 through March 2016. This study was approved by the Franciscan Health Lafayette Institutional Review Board. All procedures performed in studies involving human participants were in accordance with the ethical standards of the institutional and/or national research committee and with the 1964 Helsinki declaration and its later amendments or comparable ethical standards. Informed consent was obtained from all individual participants included in the study.

\section{Continuous Care Intervention}

Participants in the CCI underwent history and physical exam followed by laboratory testing to ensure they met inclusion and exclusion criteria (Supplementary Materials A). Upon qualifying, CCI participants received biomarker tracking tools including a cellular-connected body weight scale (BT003, Body Trace; New York, NY, USA), a finger-stick blood glucose and ketone meter (Precision Xtra, Abbott; Alameda, CA, USA), and a blood pressure cuff if hypertension was diagnosed (BP742 N, Omron Healthcare, Inc.; Lake Forest, IL, USA). Access to a web-based software application (app) was provided for biomarker reporting and monitoring, education, and communication with remote care team (via telemedicine) consisting of a health coach and medical provider (physician or nurse practitioner) for advice and medication management. Social support was provided via an online peer community. Participants in the CCI retained their primary care provider (PCP) for conditions other than metabolic disease, and care coordination between the PCP and CCI provider occurred as needed. Frequency and type of biomarker tracking were individualized on the basis of care needs and recorded by participants in the app; initial participant instructions were to weigh and measure blood $\mathrm{BHB}$ concentration daily, and to measure blood glucose one to three times daily. The remote care team monitored this information; a medical provider made medication changes as indicated by the participant-reported biomarkers (Supplementary Materials B).

Participants were provided individualized nutrition recommendations that allowed them to achieve and sustain nutritional ketosis with a goal of $0.5-3.0 \mathrm{mmol} \mathrm{L}^{-1}$ blood BHB. Participants were encouraged to report daily hunger, cravings, energy, and mood on a four-point Likert scale. These ratings and BHB concentrations were utilized to adjust nutritional guidance. With the insulin resistance characteristic of $\mathrm{T} 2 \mathrm{D}$, patients typically require total dietary carbohydrates to be restricted to less than $30 \mathrm{~g} \mathrm{day}^{-1}$ to achieve nutritional ketosis. Health coaches monitored blood BHB concentrations logged by participants and worked with participants individually to adjust dietary carbohydrate intake to a level that would allow them to achieve nutritional ketosis. Daily protein intake was initially targeted to a level of $1.5 \mathrm{~g} \mathrm{~kg}^{-1}$ of reference (i.e., medium-frame "ideal") body weight and adjusted as necessary to aid participants in achieving nutritional ketosis based on participant-logged blood BHB concentrations. Participants were coached to incorporate dietary fats to satiety. Participants were advised to consume adequate intake of omega-3 (eicosapentaenoic acid and docosahexaenoic acid) and omega-6 (linoleic acid) polyunsaturated fats [22], while it was recommended that the remainder of their intake from fat come from both monounsaturated and saturated sources. Other aspects of the diet were individually prescribed to ensure safety, effectiveness, and 
satisfaction, including consumption of 3-5 servings of non-starchy vegetables and adequate mineral and fluid intake for the ketogenic state. At onset of dietary changes, participants were advised to consume a multivitamin, 1000-2000 IU vitamin D3, and up to $1000 \mathrm{mg}$ omega-3 daily. If participants exhibited signs of magnesium depletion (e.g., muscle twitches or cramps), daily supplementation (500 mg magnesium oxide or $200 \mathrm{mg}$ magnesium chloride) was suggested. If participants exhibited headaches, constipation, or lightheadedness, adequate sodium and fluid intake was recommended. BHB concentrations were also utilized as a marker of adherence to nutritional ketosis. Behavior change strategies were utilized by the remote care team and tailored to the needs of each participant to help achieve glycemic control. Examples of techniques utilized include education of natural consequences, shaping knowledge, goal setting, self-monitoring, feedback, monitoring and reinforcement from health coach and medical provider, selfbelief, social support, relapse prevention, associations, and repetition.

Participants in the CCI self-selected how they would receive most of their education: (1) via on-site group education classes that met weekly for 12 weeks, bi-weekly for 12 weeks, and monthly for 6 months $(n=136$; CCI-onsite) or (2) via web-based, recorded educational content viewed independently through the app $(n=126$; CCI-web). Educational content was the same regardless of delivery method (Supplementary Materials C), and all other aspects of care were the same. During on-site classes, health coaches presented educational content and medical providers met with participants individually. Participants receiving web-based education could schedule visits with the CCI medical provider if desired. Apart from education delivery, both groups received remote care from health coaches.

\section{Usual Care}

Participants in the UC group were patients with diagnosed T2D who were recently referred to the local diabetes education program by their primary care physician or endocrinologist where they were counseled by registered dietitians on diabetes self-management, nutrition, and lifestyle [7]. Medical care for their T2D was provided by their primary care physician or endocrinologist. No modification to the care that they received for their T2D was made by the study. This group was observed at baseline and 1 year as reference for typical disease treatment and progression over 1 year within the same geographical, health care, and laboratory locations. UC participants attended a separate information session and informed consent was obtained followed by laboratory testing to ensure they met all inclusion and exclusion criteria. Patients were informed that the trial also had an intervention arm and could participate in that group if they chose to do so.

\section{Outcome Measures}

In-clinic vital signs and anthropometrics were obtained at baseline, 70-days (CCI only [23]), and 1-year follow-up. Height was assessed via stadiometer for calculation of body mass index. In-clinic weight for all participants was measured to the nearest $0.1 \mathrm{lb}$ (Model 750, Detecto; Webb City, MO, USA) and converted to kg. Inclinic blood pressure was obtained manually by trained staff after participants rested in a seated position for $5 \mathrm{~min}$. Adverse events were reported to the Principal Investigator and reviewed by the Institutional Review Board.

Fasted blood draws occurred at baseline, 70-days (CCI only [23]), and 1-year follow up. Blood analytes were determined via standard procedures at a Clinical Laboratory Improvement Amendment (CLIA) accredited laboratory on the day of sample collection or from stored serum (Supplementary Materials D).

\section{Statistical Analysis}

Statistical analyses were performed using JMP software (version 5.1, SAS Institute; Cary, SC, USA) for all analyses except multiple imputation, for which we used Stata software (version 11, StataCorp; College Station, TX, USA). Multiple imputation was used to estimate means 
Table 1 Baseline characteristics of the recruited sample, completers, and participants with missing data by treatment arm

\begin{tabular}{|c|c|c|c|c|c|c|c|}
\hline & \multicolumn{2}{|l|}{ All } & \multicolumn{2}{|c|}{ Completers with data } & \multicolumn{2}{|c|}{ Dropout or missing data } & \multirow{2}{*}{$\begin{array}{l}\text { Completers- } \\
\text { Dropouts } \\
\text { Mean } \pm \text { SE }\end{array}$} \\
\hline & $N$ & $\begin{array}{l}\text { Mean }(S D) \\
\text { or } \pm S E\end{array}$ & $N$ & $\begin{array}{l}\text { Mean (SD) } \\
\text { or } \pm S E\end{array}$ & $N$ & $\begin{array}{l}\text { Mean }(S D) \\
\text { or } \pm S E\end{array}$ & \\
\hline \multicolumn{8}{|l|}{ Age (years) } \\
\hline CCI-all education $^{\mathrm{a}}$ & 262 & $53.75(8.35)$ & 218 & $54.09(8.35)$ & 44 & $52.09(8.25)$ & $2.0 \pm 1.37$ \\
\hline Usual care $^{\mathrm{a}}$ & 87 & $52.33(9.52)$ & 78 & $51.71(9.62)$ & 9 & $57.78(6.85)$ & $-6.07 \pm 2.53^{*}$ \\
\hline CCI-all vs. usual care ${ }^{\mathrm{b}}$ & & $1.42 \pm 1.14$ & & $2.38 \pm 1.23^{*}$ & & $-5.69 \pm 2.6^{*}$ & \\
\hline \multicolumn{8}{|l|}{ Female (\%) } \\
\hline CCI-all education ${ }^{\mathrm{a}}$ & 262 & $66.79 \pm 2.91$ & 218 & $65.14 \pm 3.23$ & 44 & $75.0 \pm 6.53$ & $-9.86 \pm 7.28$ \\
\hline Usual care $^{\mathrm{a}}$ & 87 & $58.62 \pm 5.28$ & 78 & $60.26 \pm 5.54$ & 9 & $44.44 \pm 16.56$ & $15.81 \pm 17.47$ \\
\hline CCI-all vs. usual care ${ }^{\mathrm{b}}$ & & $8.17 \pm 6.03$ & & $4.88 \pm 6.41$ & & $30.56 \pm 17.8$ & \\
\hline \multicolumn{8}{|l|}{ African American (\%) } \\
\hline CCI-all education ${ }^{\mathrm{a}}$ & 262 & $6.87 \pm 1.56$ & 218 & $5.96 \pm 1.6$ & 44 & $11.36 \pm 4.78$ & $-5.4 \pm 5.05$ \\
\hline Usual care $^{\mathrm{a}}$ & 87 & $0.0 \pm 0.0$ & 78 & $0.0 \pm 0.0$ & 9 & $0.0 \pm 0.0$ & $0.0 \pm 0.0$ \\
\hline CCI-all vs. usual care ${ }^{\mathrm{b}}$ & & $6.87 \pm 1.56 \$$ & & $5.96 \pm 1.6 t$ & & $11.36 \pm 4.78^{*}$ & \\
\hline \multicolumn{8}{|l|}{ Years with type 2 diabetes } \\
\hline CCI-all education $^{\mathrm{a}}$ & 261 & $8.44(7.22)$ & 217 & $8.4(7.28)$ & 44 & $8.61(6.97)$ & $-0.21 \pm 1.16$ \\
\hline Usual care $^{\mathrm{a}}$ & 71 & $7.85(7.32)$ & 71 & $7.85(7.32)$ & & Not collected & \\
\hline CCI-all vs. usual care ${ }^{\mathrm{b}}$ & & $0.59(0.9)$ & & $0.56 \pm 1.0$ & & & \\
\hline \multicolumn{8}{|c|}{ Beta-hydroxybutyrate $\left(\mathrm{mmol} \mathrm{L}^{-1}\right)$} \\
\hline CCI-all education ${ }^{\mathrm{a}}$ & 248 & $0.17(0.15)$ & 186 & $0.17(0.15)$ & 62 & $0.19(0.16)$ & $-0.02 \pm 0.02$ \\
\hline Usual care $^{\mathrm{a}}$ & 79 & $0.15(0.13)$ & 59 & $0.14(0.12)$ & 20 & $0.17(0.15)$ & $-0.03 \pm 0.03$ \\
\hline CCI-all vs. usual care ${ }^{\mathrm{b}}$ & & $0.02 \pm 0.02$ & & $0.02 \pm 0.02$ & & $0.02 \pm 0.04$ & \\
\hline \multicolumn{8}{|c|}{ Hemoglobin $\mathrm{A}_{\mathrm{lc}}\left(\mathrm{mmol} \mathrm{mol}{ }^{-1}\right)$} \\
\hline CCI-all education ${ }^{a}$ & 262 & $59.55(16.4)$ & 204 & $58.35(15.3)$ & 58 & $63.49(19.57)$ & $-28.66 \pm 2.73$ \\
\hline Usual care $^{\mathrm{a}}$ & 87 & $59.99(19.24)$ & 72 & $61.08(19.89)$ & 15 & $54.52(14.87)$ & $-16.97 \pm 4.48$ \\
\hline CCI-all vs. usual care ${ }^{\mathrm{b}}$ & & $-0.44 \pm 2.3$ & & $-2.73 \pm 2.62$ & & $8.96 \pm 4.59^{*}$ & \\
\hline \multicolumn{8}{|l|}{ Hemoglobin $A_{1 c}(\%)$} \\
\hline CCI-all education ${ }^{\mathrm{a}}$ & 262 & $7.60(1.50)$ & 204 & $7.49(1.4)$ & 58 & $7.96(1.79)$ & $-0.47 \pm 0.25$ \\
\hline Usual care $^{\mathrm{a}}$ & 87 & $7.64(1.76)$ & 72 & $7.74(1.82)$ & 15 & $7.14(1.36)$ & $0.60 \pm 0.41$ \\
\hline CCI-all vs. usual care ${ }^{\mathrm{b}}$ & & $-0.04 \pm 0.21$ & & $-0.25 \pm 0.24$ & & $0.82 \pm 0.42^{*}$ & \\
\hline \multicolumn{8}{|c|}{ Fasting glucose $\left(\mathrm{mmol} \mathrm{L}^{-1}\right)$} \\
\hline CCI-all education ${ }^{\mathrm{a}}$ & 258 & $8.92(3.41)$ & 202 & $8.8(3.28)$ & 56 & $9.36(3.83)$ & $-0.55 \pm 0.56$ \\
\hline Usual care $^{\mathrm{a}}$ & 86 & $8.67(4.03)$ & 71 & $8.71(3.96)$ & 15 & $8.5(4.5)$ & $0.21 \pm 1.25$ \\
\hline CCI-all vs. usual care ${ }^{\mathrm{b}}$ & & $0.25 \pm 0.48$ & & $0.1 \pm 0.52$ & & $0.86 \pm 1.27$ & \\
\hline
\end{tabular}


Table 1 continued

\begin{tabular}{|c|c|c|c|c|c|c|c|}
\hline & \multicolumn{2}{|l|}{ All } & \multicolumn{2}{|c|}{ Completers with data } & \multicolumn{2}{|c|}{ Dropout or missing data } & \multirow{2}{*}{$\begin{array}{l}\text { Completers- } \\
\text { Dropouts } \\
\text { Mean } \pm \text { SE }\end{array}$} \\
\hline & $N$ & $\begin{array}{l}\text { Mean (SD) } \\
\text { or } \pm S E\end{array}$ & $N$ & $\begin{array}{l}\text { Mean (SD) } \\
\text { or } \pm S E\end{array}$ & $N$ & $\begin{array}{l}\text { Mean (SD) } \\
\text { or } \pm S E\end{array}$ & \\
\hline \multicolumn{8}{|l|}{ Insulin all $\left(\mathrm{pmol} \mathrm{L}^{-1}\right)$} \\
\hline CCI-all education ${ }^{\mathrm{a}}$ & 248 & $198.35(165.85)$ & 186 & $197.65(167.17)$ & 62 & $200.5(163.21)$ & $-2.85 \pm 24.1$ \\
\hline Usual care $^{\mathrm{a}}$ & 79 & $202.17(172.58)$ & 59 & $206.68(187.93)$ & 20 & $188.77(119.18)$ & $17.99 \pm 36.18$ \\
\hline CCI-all vs. usual care ${ }^{\mathrm{b}}$ & & $-3.82 \pm 22.09$ & & $-9.1 \pm 27.36$ & & $11.74 \pm 33.75$ & \\
\hline \multicolumn{8}{|l|}{ C-peptide $\left(\mathrm{nmol} \mathrm{L}^{-1}\right)$} \\
\hline CCI-all education ${ }^{a}$ & 247 & $1.45(0.71)$ & 185 & $1.47(0.72)$ & 62 & $1.39(0.69)$ & $0.07 \pm 0.1$ \\
\hline Usual care $^{\mathrm{a}}$ & 79 & $1.38(0.82)$ & 59 & $1.35(0.82)$ & 20 & $1.49(0.84)$ & $-0.14 \pm 0.22$ \\
\hline CCI-all vs. usual care ${ }^{b}$ & & $0.07 \pm 0.1$ & & $0.12 \pm 0.12$ & & $-0.09 \pm 0.21$ & \\
\hline \multicolumn{8}{|c|}{ HOMA-IR (insulin derived), all } \\
\hline CCI-all education ${ }^{\mathrm{a}}$ & 244 & $11.8(13.14)$ & 179 & $11.19(12.75)$ & 65 & $13.48(14.12)$ & $-2.3 \pm 1.99$ \\
\hline Usual care $^{\mathrm{a}}$ & 78 & $10.64(9.12)$ & 56 & $11.31(10.05)$ & 22 & $8.94(6.03)$ & $2.36 \pm 1.86$ \\
\hline CCI-all vs. usual care ${ }^{b}$ & & $1.16 \pm 1.33$ & & $-0.12 \pm 1.65$ & & $4.54 \pm 2.17$ & \\
\hline \multicolumn{8}{|c|}{ HOMA-IR (insulin derived), excluding exogenous users } \\
\hline CCI-all education ${ }^{a}$ & 172 & $11.77(13.87)$ & 129 & $11.00(13.53)$ & 43 & $14.09(14.76)$ & $-3.08 \pm 2.55$ \\
\hline Usual care $^{\mathrm{a}}$ & 43 & $9.40(8.25)$ & 25 & $9.36(9.39)$ & 18 & $9.45(6.61)$ & $-0.09 \pm 2.44$ \\
\hline CCI-all vs. usual care ${ }^{\mathrm{b}}$ & & $2.37 \pm 1.64$ & & $1.64 \pm 2.22$ & & $4.63 \pm 2.74$ & \\
\hline \multicolumn{8}{|c|}{ HOMA-IR (C-peptide derived) } \\
\hline CCI-all education $^{\mathrm{a}}$ & 239 & $11.52(7.15)$ & 170 & $11.44(6.26)$ & 69 & $11.72(9.04)$ & $-0.28 \pm 1.19$ \\
\hline Usual care $^{\mathrm{a}}$ & 72 & $11.16(7.26)$ & 47 & $10.56(7.70)$ & 25 & $12.29(6.33)$ & $-1.73 \pm 1.69$ \\
\hline CCI-all vs. usual care ${ }^{\mathrm{b}}$ & & $0.36 \pm 0.97$ & & $0.88 \pm 1.22$ & & $-0.56 \pm 1.67$ & \\
\hline \multicolumn{8}{|l|}{ Weight-clinic (kg) } \\
\hline CCI-all education ${ }^{\mathrm{a}}$ & 257 & $116.51(25.94)$ & 184 & $115.42(24.62)$ & 73 & $119.25(29.01)$ & $-3.83 \pm 3.85$ \\
\hline Usual care $^{\mathrm{a}}$ & 83 & $105.63(22.15)$ & 69 & $106.79(22.18)$ & 14 & $99.94(21.86)$ & $6.84 \pm 6.42$ \\
\hline CCI-all vs. usual care ${ }^{\mathrm{b}}$ & & $10.87 \pm 2.92 \S$ & & $8.63 \pm 3.23 \dagger$ & & $19.3 \pm 6.76 \dagger$ & \\
\hline \multicolumn{8}{|l|}{ BMI $\left(\mathrm{kg} \mathrm{m}^{-2}\right)$} \\
\hline CCI-all education ${ }^{\mathrm{a}}$ & 257 & $40.43(8.81)$ & 184 & $39.87(7.88)$ & 73 & $41.82(10.75)$ & $-1.94 \pm 1.39$ \\
\hline Usual care $^{\mathrm{a}}$ & 83 & $36.72(7.26)$ & 69 & $37.14(7.62)$ & 14 & $34.66(4.8)$ & $2.48 \pm 1.58$ \\
\hline CCI-all vs. usual care ${ }^{b}$ & & $3.7 \pm 0.97 t$ & & $2.73 \pm 1.09 \dagger$ & & $7.15 \pm 1.8 \S$ & \\
\hline \multicolumn{8}{|c|}{ Systolic blood pressure $(\mathrm{mmHg})$} \\
\hline CCI-all education ${ }^{\mathrm{a}}$ & 260 & $131.94(14.09)$ & 187 & $132.51(14.54)$ & 73 & $130.47(12.84)$ & $2.05 \pm 1.84$ \\
\hline Usual care $^{\mathrm{a}}$ & 79 & $129.8(13.61)$ & 67 & $128.72(12.65)$ & 12 & $135.83(17.49)$ & $-7.12 \pm 5.28$ \\
\hline CCI-all vs. usual care ${ }^{\mathrm{b}}$ & & $2.14 \pm 1.76$ & & $3.8 \pm 1.88^{*}$ & & $-5.37 \pm 5.27$ & \\
\hline
\end{tabular}


Table 1 continued

\begin{tabular}{|c|c|c|c|c|c|c|}
\hline All & & & leters with data & Dr & t or missing data & Completers- \\
\hline$N$ & $\begin{array}{l}\text { Mean (SD) } \\
\text { or } \pm S E\end{array}$ & $N$ & $\begin{array}{l}\text { Mean (SD) } \\
\text { or } \pm S E\end{array}$ & $N$ & $\begin{array}{l}\text { Mean (SD) } \\
\text { or } \pm S E\end{array}$ & Mean $\pm S E$ \\
\hline
\end{tabular}

Diastolic blood pressure (mmHg)

Usual care

$260 \quad 82.09(8.25)$

$187 \quad 81.59(8.05)$

73

83.37 (8.67)

$-1.78 \pm 1.17$

sual care ${ }^{a}$

$79 \quad 82.0(8.93)$

67

$81.1(8.07)$

12

$87.0(11.95)$

$-5.9 \pm 3.59$

CCI-all vs. usual care ${ }^{\mathrm{b}}$

$0.09 \pm 1.13$

$0.49 \pm 1.15$

$-3.63 \pm 3.6$

Total cholesterol $\left(\mathrm{mmol} \mathrm{L}^{-1}\right)$

CCI-all education ${ }^{\mathrm{a}}$

$247 \quad 4.76(1.07)$

$1864.68(1.03)$

61

$4.99(1.15)$

$-0.31 \pm 0.17$

Usual care $^{\mathrm{a}}$

$79 \quad 4.76(1.19)$

59

$4.72(1.26)$

$4.88(0.93)$

$-0.16 \pm 0.27$

CCI-all vs. usual care ${ }^{\mathrm{b}}$

$-0.0 \pm 0.15$

$-0.04 \pm 0.18$

$0.11 \pm 0.26$

LDL-cholesterol $\left(\mathrm{mmol} \mathrm{L}^{-1}\right)$

CCI-all education ${ }^{\mathrm{a}}$

$232 \quad 102.51(32.89)$

$172 \quad 100.08(32.56)$

60

$109.47(33.13)$

$-9.39 \pm 4.94$

Usual care $^{\mathrm{a}}$

$70 \quad 101.50(36.16)$

48

100.38 (37.93)

22

103.95 (32.67)

$-3.58 \pm 8.86$

CCI-all vs. usual care ${ }^{b}$

$1.01 \pm 4.83$

$-0.29 \pm 6.01$

$5.51 \pm 8.17$

Apo B $\left(\mathrm{g} \mathrm{L}^{-1}\right)$

CCI-all education

$248 \quad 1.05(0.29)$

$186 \quad 1.03(0.28)$

62

$1.1(0.31)$

$-0.06 \pm 0.04$

Usual care $^{\mathrm{a}}$

$79 \quad 1.07(0.28)$

$59 \quad 1.06(0.3)$

20

$1.11(0.24)$

$-0.05 \pm 0.07$

CCI-all vs. usual care ${ }^{b}$

$-0.02 \pm 0.04$

$-0.02 \pm 0.04$

$-0.01 \pm 0.07$

HDL-C $\left(\mathrm{mmol} \mathrm{L}^{-1}\right)$

CCI-all education ${ }^{\mathrm{a}}$

$247 \quad 1.09(0.35)$

$186 \quad 1.1(0.36)$

61

$1.08(0.32)$

$0.02 \pm 0.05$

Usual care $^{\mathrm{a}}$

$79 \quad 0.97(0.29)$

59

$0.96(0.29)$

20

$1.02(0.29)$

$-0.06 \pm 0.08$

CCI-all vs. usual care ${ }^{b}$

$0.12 \pm 0.04 \dagger$

$0.14 \pm 0.05 \dagger$

$0.06 \pm 0.08$

Triglycerides $\left(\mathrm{mmol} \mathrm{L}^{-1}\right)$

CCI-all education

$247 \quad 2.23(1.62)$

$186 \quad 2.27(1.73)$

61

$2.11(1.25)$

$0.15 \pm 0.2$

Usual care $^{\mathrm{a}}$

$79 \quad 3.2(4.53)$

$59 \quad 3.36(5.17)$

20

$2.72(1.56)$

$0.64 \pm 0.76$

CCI-all vs. usual care ${ }^{\mathrm{b}}$

$-0.97 \pm 0.52^{*}$

$-1.09 \pm 0.68$

$-0.61 \pm 0.38^{*}$

Total/HDL-cholesterol

CCI-all education ${ }^{\mathrm{a}}$

Usual care ${ }^{\mathrm{a}}$

$$
247 \quad 4.72(1.7)
$$

$79 \quad 5.37(2.42)$

CCI-all vs. usual care ${ }^{\mathrm{b}}$

$-0.65 \pm 0.29^{*}$

hsC-reactive protein $\left(\mathrm{nmol} \mathrm{L}^{-1}\right)$

CCI-all education ${ }^{\mathrm{a}}$

249

Usual care ${ }^{\mathrm{a}}$

$85 \quad 84.67(82.1)$

$-3.24 \pm 12.48$

CCI-all vs. usual care ${ }^{\mathrm{b}}$
$186 \quad 4.65(1.72)$

$59 \quad 5.44(2.63)$

$-0.79 \pm 0.36^{*}$

61

$4.93(1.65)$

$5.17(1.72)$

$-0.24 \pm 0.44$

$193 \quad 85.62(153.05)$

56

$66.76(62.1)$

$86.95(86.95)$

15

$-1.33 \pm 15.05$
$73.81(73.81)$

$-7.05 \pm 18.19$
$-0.28 \pm 0.25$

$0.27 \pm 0.52$

$18.86 \pm 13.81$

$13.14 \pm 19.14$

$-3.24 \pm 12.48$

-

(

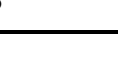


Table 1 continued

\begin{tabular}{|c|c|c|c|c|c|c|c|}
\hline & \multicolumn{2}{|l|}{ All } & \multicolumn{2}{|c|}{ Completers with data } & \multicolumn{2}{|c|}{ Dropout or missing data } & \multirow{2}{*}{$\begin{array}{l}\text { Completers- } \\
\text { Dropouts } \\
\text { Mean } \pm \text { SE }\end{array}$} \\
\hline & $N$ & $\begin{array}{l}\text { Mean (SD) } \\
\text { or } \pm S E\end{array}$ & $N$ & $\begin{array}{l}\text { Mean (SD) } \\
\text { or } \pm S E\end{array}$ & $N$ & $\begin{array}{l}\text { Mean (SD) } \\
\text { or } \pm S E\end{array}$ & \\
\hline \multicolumn{8}{|l|}{$\operatorname{ALT}\left(\mu \mathrm{kat} \mathrm{L}^{-1}\right)$} \\
\hline CCI-all education ${ }^{\mathrm{a}}$ & 257 & $0.51(0.38)$ & 201 & $0.52(0.41)$ & 56 & $0.47(0.27)$ & $0.05 \pm 0.05$ \\
\hline Usual care $^{\mathrm{a}}$ & 86 & $0.46(0.33)$ & 71 & $0.45(0.34)$ & 15 & $0.51(0.29)$ & $-0.05 \pm 0.09$ \\
\hline CCI-all vs. usual care ${ }^{\mathrm{b}}$ & & $0.05 \pm 0.04$ & & $0.07 \pm 0.05$ & & $-0.04 \pm 0.08$ & \\
\hline \multicolumn{8}{|l|}{$\operatorname{AST}\left(\mu\right.$ kat $\left.L^{-1}\right)$} \\
\hline CCI-all education ${ }^{a}$ & 257 & $0.4(0.25)$ & 201 & $0.41(0.28)$ & 56 & $0.36(0.15)$ & $0.04 \pm 0.03$ \\
\hline Usual care $^{\mathrm{a}}$ & 86 & $0.4(0.32)$ & 71 & $0.39(0.35)$ & 15 & $0.42(0.16)$ & $-0.03 \pm 0.06$ \\
\hline CCI-all vs. usual care ${ }^{\mathrm{b}}$ & & $-0.0 \pm 0.04$ & & $0.01 \pm 0.05$ & & $-0.06 \pm 0.05$ & \\
\hline \multicolumn{8}{|c|}{ Alkaline phosphatase $\left(\mu \mathrm{kat}^{-1}\right)$} \\
\hline CCI-all education ${ }^{\mathrm{a}}$ & 256 & $1.24(0.37)$ & 200 & $1.24(0.37)$ & 56 & $1.23(0.36)$ & $0.01 \pm 0.05$ \\
\hline Usual care $^{\mathrm{a}}$ & 86 & $1.29(0.44)$ & 71 & $1.31(0.45)$ & 15 & $1.22(0.38)$ & $0.09 \pm 0.11$ \\
\hline CCI-all vs. usual care ${ }^{\mathrm{b}}$ & & $-0.05 \pm 0.05$ & & $-0.07 \pm 0.06$ & & $0.01 \pm 0.11$ & \\
\hline \multicolumn{8}{|c|}{ Serum creatinine $\left(\mu \mathrm{mol} \mathrm{L}{ }^{-1}\right)$} \\
\hline CCI-all education ${ }^{\mathrm{a}}$ & 258 & $77.79(21.22)$ & 202 & $77.79(20.33)$ & 56 & $81.33(24.75)$ & $-3.54 \pm 3.54$ \\
\hline Usual care $^{\mathrm{a}}$ & 86 & $80.44(22.1)$ & 71 & $78.68(20.33)$ & 15 & $86.63(25.64)$ & $-7.07 \pm 7.07$ \\
\hline CCI-all vs. usual care ${ }^{\mathrm{b}}$ & & $-1.77 \pm 2.65$ & & $-1.77 \pm 2.65$ & & $-5.3 \pm 7.07$ & \\
\hline \multicolumn{8}{|l|}{ BUN $\left(\mathrm{mmol} \mathrm{L}^{-1}\right)$} \\
\hline CCI-all education ${ }^{\mathrm{a}}$ & 258 & $6.03(2.34)$ & 202 & $6.06(2.15)$ & 56 & $5.9(2.96)$ & $0.16 \pm 0.42$ \\
\hline Usual care $^{\mathrm{a}}$ & 86 & $5.73(2.23)$ & 71 & $5.59(1.86)$ & 15 & $6.38(3.52)$ & $-0.79 \pm 0.94$ \\
\hline CCI-all vs. usual care ${ }^{\mathrm{b}}$ & & $0.3 \pm 0.28$ & & $0.47 \pm 0.27$ & & $-0.47 \pm 0.99$ & \\
\hline \multicolumn{8}{|l|}{$\mathrm{eGFR}\left(\mathrm{mL} \mathrm{s}^{-1} \mathrm{~m}^{-2}\right)$} \\
\hline CCI-all education $^{\mathrm{a}}$ & 258 & $1.34(0.23)$ & 202 & $1.35(0.22)$ & 56 & $1.33(0.25)$ & $0.02 \pm 0.04$ \\
\hline Usual care $^{\mathrm{a}}$ & 86 & $1.32(0.23)$ & 71 & $1.34(0.22)$ & 15 & $1.26(0.28)$ & $0.08 \pm 0.08$ \\
\hline CCI-all vs. usual care ${ }^{b}$ & & $0.02 \pm 0.03$ & & $0.02 \pm 0.03$ & & $0.03 \pm 0.08$ & \\
\hline \multicolumn{8}{|l|}{ Anion gap $\left(\mathrm{mmol} \mathrm{L}^{-1}\right)$} \\
\hline CCI-all education $^{a}$ & 257 & $6.83(1.67)$ & 201 & $6.79(1.7)$ & 56 & $6.98(1.53)$ & $-0.19 \pm 0.24$ \\
\hline Usual care $^{\mathrm{a}}$ & 86 & $6.93(1.82)$ & 71 & $6.92(1.82)$ & 15 & $7.0(1.89)$ & $-0.08 \pm 0.53$ \\
\hline CCI-all vs. usual care ${ }^{b}$ & & $-0.1 \pm 0.22$ & & $-0.12 \pm 0.25$ & & $-0.02 \pm 0.53$ & \\
\hline \multicolumn{8}{|l|}{ Uric acid $\left(\mu \mathrm{mo} \mathrm{L}^{-1}\right)$} \\
\hline CCI-all education $^{\mathrm{a}}$ & 261 & $347.99(86.85)$ & 202 & $348.58(86.25)$ & 59 & $346.2(89.82)$ & $2.38 \pm 13.09$ \\
\hline Usual care $^{\mathrm{a}}$ & 85 & $333.12(87.44)$ & 71 & $330.74(85.66)$ & 14 & $345.01(98.75)$ & $-14.28 \pm 28.55$ \\
\hline CCI-all vs. usual care ${ }^{\mathrm{b}}$ & & $14.87 \pm 10.71$ & & $17.25 \pm 11.9$ & & $1.19 \pm 29.15$ & \\
\hline
\end{tabular}


Table 1 continued

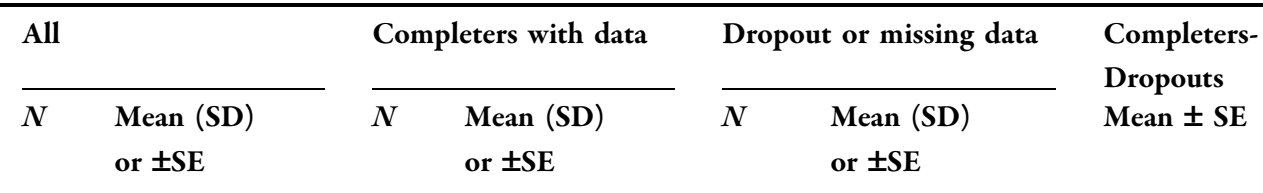

TSH $\left(\mathrm{mIU} \mathrm{L}^{-1}\right)$

CCI-all education ${ }^{\mathrm{a}}$

Usual care $^{\mathrm{a}}$

$$
259
$$

$2.32(1.74)$

$85 \quad 1.97(1.16)$

$0.36 \pm 0.17^{*}$

$\begin{array}{ll}200 & 2.31(1.79) \\ 70 & 2.09(1.16) \\ & 0.21 \pm 0.19\end{array}$

59

$2.38(1.55)$

15

1.38 (1.03)

$1.0 \pm 0.33 \dagger$

Free T4 $\left(\mathrm{pmol} \mathrm{L}^{-1}\right)$

CCI-all education ${ }^{a}$

Usual care $^{\mathrm{a}}$

CCI-all vs. usual care ${ }^{\mathrm{b}}$

Any diabetes medication, excluding metformin (\%)

\section{CCI-all education ${ }^{a}$}

Usual care $^{\mathrm{a}}$

CCI-all vs. usual care ${ }^{\mathrm{b}}$

Sulfonylurea (\%)

CCI-all education ${ }^{\mathrm{a}}$

Usual care $^{\mathrm{a}}$

CCI-all vs. usual care ${ }^{\mathrm{b}}$

Insulin (\%)

CCI-all education ${ }^{\mathrm{a}}$

Usual care ${ }^{\mathrm{a}}$

CCI-all vs. usual care ${ }^{\mathrm{b}}$

Thiazolidinedione (\%)

CCI-all education ${ }^{\mathrm{a}}$

Usual care $^{\mathrm{a}}$

CCI-all vs. usual care ${ }^{b}$

SGLT-2 (\%)

CCI-all education ${ }^{\mathrm{a}}$

Usual care $^{\mathrm{a}}$

CCI-all vs. usual care ${ }^{\mathrm{b}}$

DPP-4 (\%)

CCI-all education ${ }^{\mathrm{a}}$

Usual care $^{\mathrm{a}}$

CCI-all vs. usual care ${ }^{\mathrm{b}}$
260

$11.84(2.19)$

86

$11.33(3.73)$

$0.51 \pm 0.39$

$$
\begin{array}{ll}
262 & 56.87 \pm 3.06 \\
87 & 66.67 \pm 5.05 \\
& -9.80 \pm 5.91
\end{array}
$$

$202 \quad 11.84(2.32)$

$71 \quad 11.33(3.86)$

$0.51 \pm 0.51$

58

11.58 (2.19)

$0.26 \pm 0.39$

15

$10.94(2.32)$

$0.64 \pm 0.64$

$0.39 \pm 0.77$

$218 \quad 55.50 \pm 3.37 \quad 44$

$63.64 \pm 7.25$

$-8.13 \pm 8.00$

$73 \quad 68.49 \pm 5.44$

14

$57.14 \pm 13.23$

$11.35 \pm 14.32$

$-12.99 \pm 6.39^{*}$

$6.49 \pm 15.08$

$$
\begin{array}{ll}
262 & 23.66 \pm 2.63 \\
87 & 24.14 \pm 4.59 \\
& -0.48 \pm 5.29
\end{array}
$$

$$
\begin{array}{ll}
218 & 24.31 \pm 2.91 \\
73 & 23.29 \pm 4.95 \\
& 1.02 \pm 5.74
\end{array}
$$

$20.45 \pm 6.08$

$28.57 \pm 12.07$

\begin{tabular}{|c|c|c|c|}
\hline 262 & $1.53 \pm 0.76$ & 218 & $1.83 \pm 0.91$ \\
\hline 87 & $1.15 \pm 1.14$ & 73 & $1.37 \pm 1.36$ \\
\hline & $0.38 \pm 1.37$ & & $0.46 \pm 1.64$ \\
\hline
\end{tabular}

$-8.12 \pm 13.52$

$$
\begin{array}{ll}
262 & 29.77 \pm 2.82 \\
87 & 45.98 \pm 5.34 \\
& -16.21 \pm 6.04 \dagger
\end{array}
$$

$$
\begin{array}{ll}
218 & 28.44 \pm 3.06 \\
78 & 50.0 \pm 5.66 \\
& -21.56 \pm 6.43 \ddagger
\end{array}
$$$$
44
$$$$
36.36 \pm 7.25
$$$$
-7.92 \pm 7.87
$$$$
9
$$$$
11.11 \pm 10.48
$$$$
25.25 \pm 12.74^{*}
$$

$38.89(1.91) \ddagger$

44

$0.0 \pm 0.0$

$1.83 \pm 0.91^{*}$

14

$0.0 \pm 0.0$

$1.37 \pm 1.36$

$0.0 \pm 0.0$

$\begin{array}{ll}262 & 10.31 \pm 1.88 \\ 87 & 13.79 \pm 3.7 \\ & -3.48 \pm 4.15\end{array}$
$218 \quad 10.55 \pm 2.08$
$73 \quad 15.07 \pm 4.19$
$-4.52 \pm 4.68$

44

$9.09 \pm 4.33$

$1.46 \pm 4.81$

14

$7.14 \pm 6.88$

$7.93 \pm 8.06$

$1.95 \pm 8.13^{*}$

$\begin{array}{ll}218 & 10.09 \pm 2.04 \\ 73 & 8.22 \pm 3.21 \\ & 1.87 \pm 3.81\end{array}$

44

$9.09 \pm 4.33$

$1.0 \pm 4.79$

14

$7.14 \pm 6.88$

$1.08 \pm 7.60$ 
Table 1 continued

\begin{tabular}{|c|c|c|c|c|c|c|c|}
\hline & \multicolumn{2}{|l|}{ All } & \multicolumn{2}{|c|}{ Completers with data } & \multicolumn{2}{|c|}{ Dropout or missing data } & \multirow{2}{*}{$\begin{array}{l}\text { Completers- } \\
\text { Dropouts } \\
\text { Mean } \pm S E\end{array}$} \\
\hline & $N$ & $\begin{array}{l}\text { Mean (SD) } \\
\text { or } \pm S E\end{array}$ & $N$ & $\begin{array}{l}\text { Mean (SD) } \\
\text { or } \pm S E\end{array}$ & $N$ & $\begin{array}{l}\text { Mean }(S D) \\
\text { or } \pm S E\end{array}$ & \\
\hline \multicolumn{8}{|l|}{ GLP-1 (\%) } \\
\hline CCI-all education ${ }^{\mathrm{a}}$ & 262 & $13.36 \pm 2.1$ & 218 & $12.84 \pm 2.27$ & 44 & $15.91 \pm 5.51$ & $-3.07 \pm 5.96$ \\
\hline Usual care $^{\mathrm{a}}$ & 87 & $14.94 \pm 3.82$ & 73 & $16.44 \pm 4.34$ & 14 & $7.14 \pm 6.88$ & $9.30 \pm 8.14$ \\
\hline CCI-all vs. usual care ${ }^{b}$ & & $-1.58 \pm 4.36$ & & $-3.59 \pm 4.89$ & & $8.77 \pm 8.82$ & \\
\hline \multicolumn{8}{|l|}{ Metformin (\%) } \\
\hline CCI-all education ${ }^{\mathrm{a}}$ & 262 & $71.37 \pm 2.79$ & 218 & $71.56 \pm 3.06$ & 44 & $70.45 \pm 6.88$ & $1.11 \pm 7.53$ \\
\hline Usual care $^{\mathrm{a}}$ & 87 & $60.92 \pm 5.23$ & 73 & $61.64 \pm 5.69$ & 14 & $57.14 \pm 13.23$ & $4.50 \pm 14.40$ \\
\hline CCI-all vs. usual care ${ }^{\mathrm{b}}$ & & $10.45 \pm 5.93$ & & $9.92 \pm 6.46$ & & $13.31 \pm 14.91$ & \\
\hline
\end{tabular}

See Table S1 (electronic supplemental material) for CCI-web, CCI-onsite, and additional comparisons

${ }^{a}$ Mean and standard deviations for continuous variables, percentages and standard errors for categorical variables

b Difference between means or percentages \pm 1 standard error of the difference. Significant baseline difference between means or percentages at $0.05>P \geq 0.01\left(^{*}\right) ; 0.01>P \geq 0.001(\dagger) ; 0.001>P \geq 0.0001(\$)$; and $P<0.0001$ (\$)

and standard errors that include the variability between imputations. Missing values were estimated from 700 imputations from multivariate normal regression. The number of missing data points for each measure can be determined from the difference between all participants and completers in Tables 1 and S1. Across all biomarkers, $4 \%$ of baseline values and $24 \%$ of 1-year values were missing (due to dropout, incalculable values, or inability to procure timely samples) and thus imputed to conduct the intention-to-treat analysis. Two-sample $t$ tests were used to test whether baseline differences and differences between 1-year biomarker changes were significant. Within-group changes were tested using paired $t$ test and analysis of covariance (ANCOVA) when adjusted for baseline covariates (sex, age, baseline BMI, insulin use versus non-use, and AfricanAmerican race). Although tables present triglyceride and hsCRP summary statistics in clinical units, significance levels were obtained from log-transformed values to reduce skewness. For completer analysis, percent change was calculated as the mean difference (Table 2) divided by the mean baseline value (Table 1 ). Significant changes in medication use and the proportion of patients with $\mathrm{HbA}_{1 \mathrm{c}}$ at least $48 \mathrm{mmol} \mathrm{mol}^{-1}(\geq 6.5 \%)$ were tested using McNemar test with continuity correction in completers, and linear regression of the changes in the dichotomous states when missing outcome data were imputed. Standard deviations are presented within parentheses and standard errors following " \pm ". Nominal significance levels $(P)$ are presented in tables; however, a significance level of $P<0.0017$ ensures simultaneous significance at $P<0.05$ with Bonferroni adjustment for the 30 variables examined. Results presented are intention-to-treat analyses (all), where missing values were estimated by imputation, unless otherwise noted. Participants who withdrew or lacked biomarkers at 1 year were not included in the analyses of completers.

\section{RESULTS}

\section{Participant Characteristics}

Table 1 presents baseline characteristics of the 262 CCI and 87 UC participants. At baseline, $88 \%$ of CCI participants were prescribed 


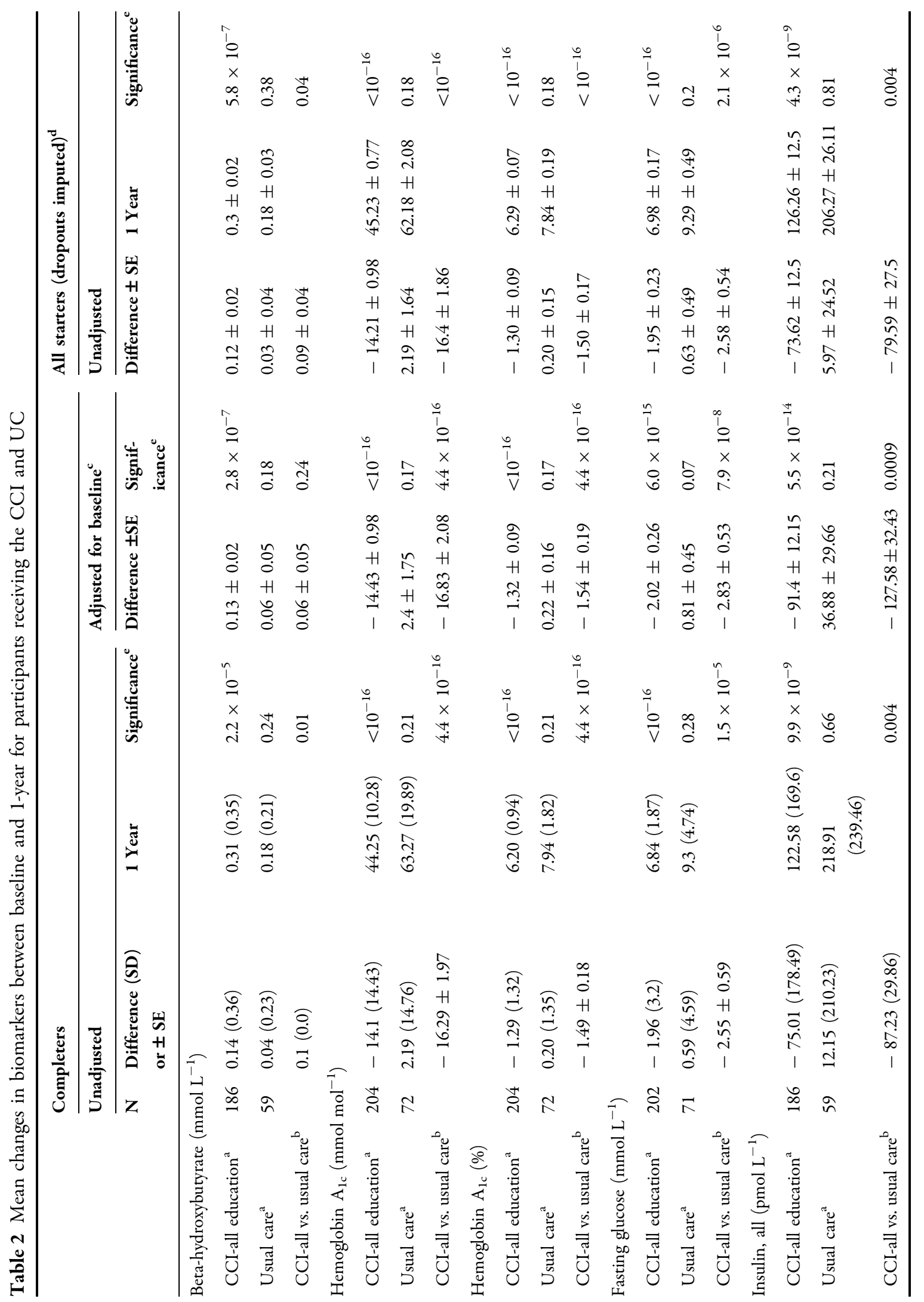




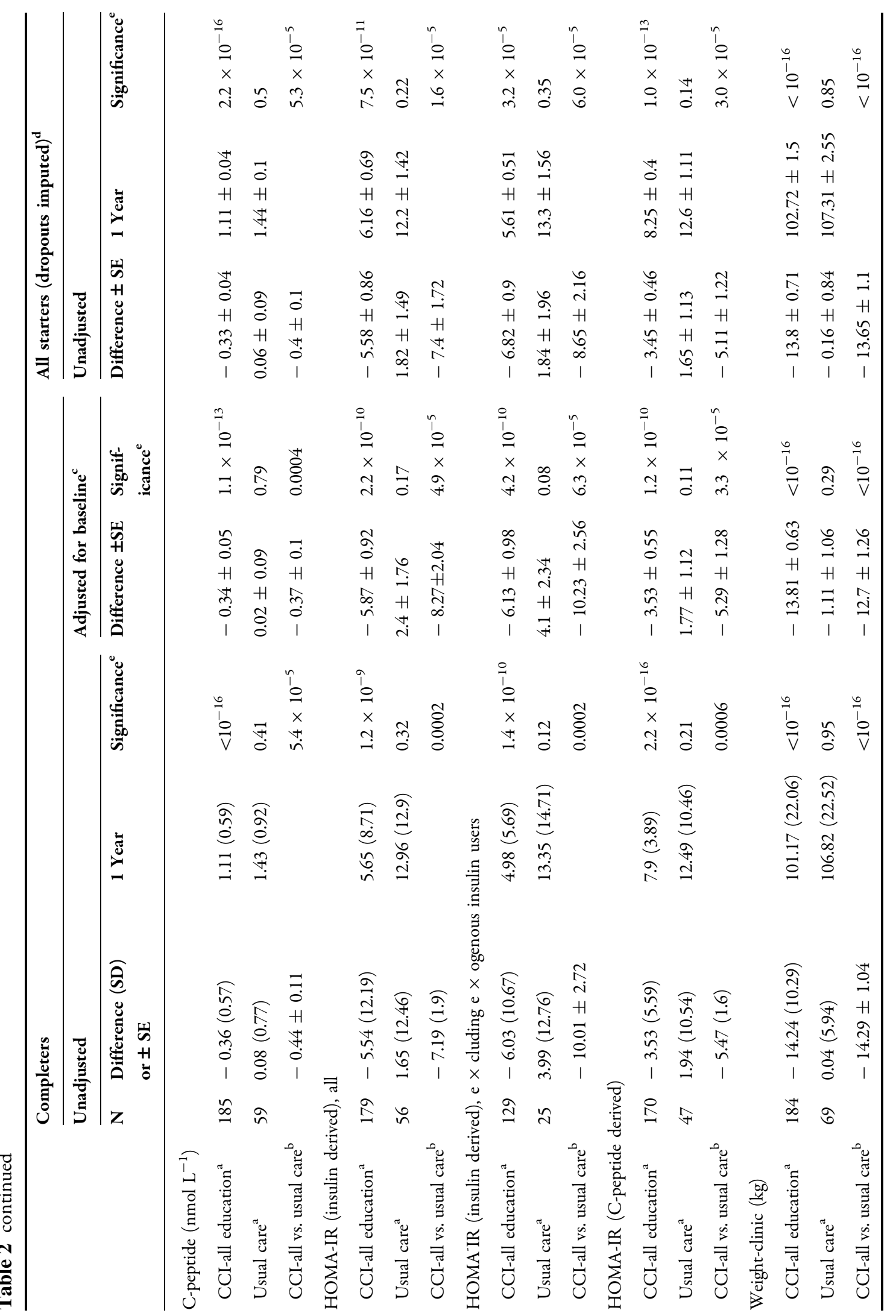




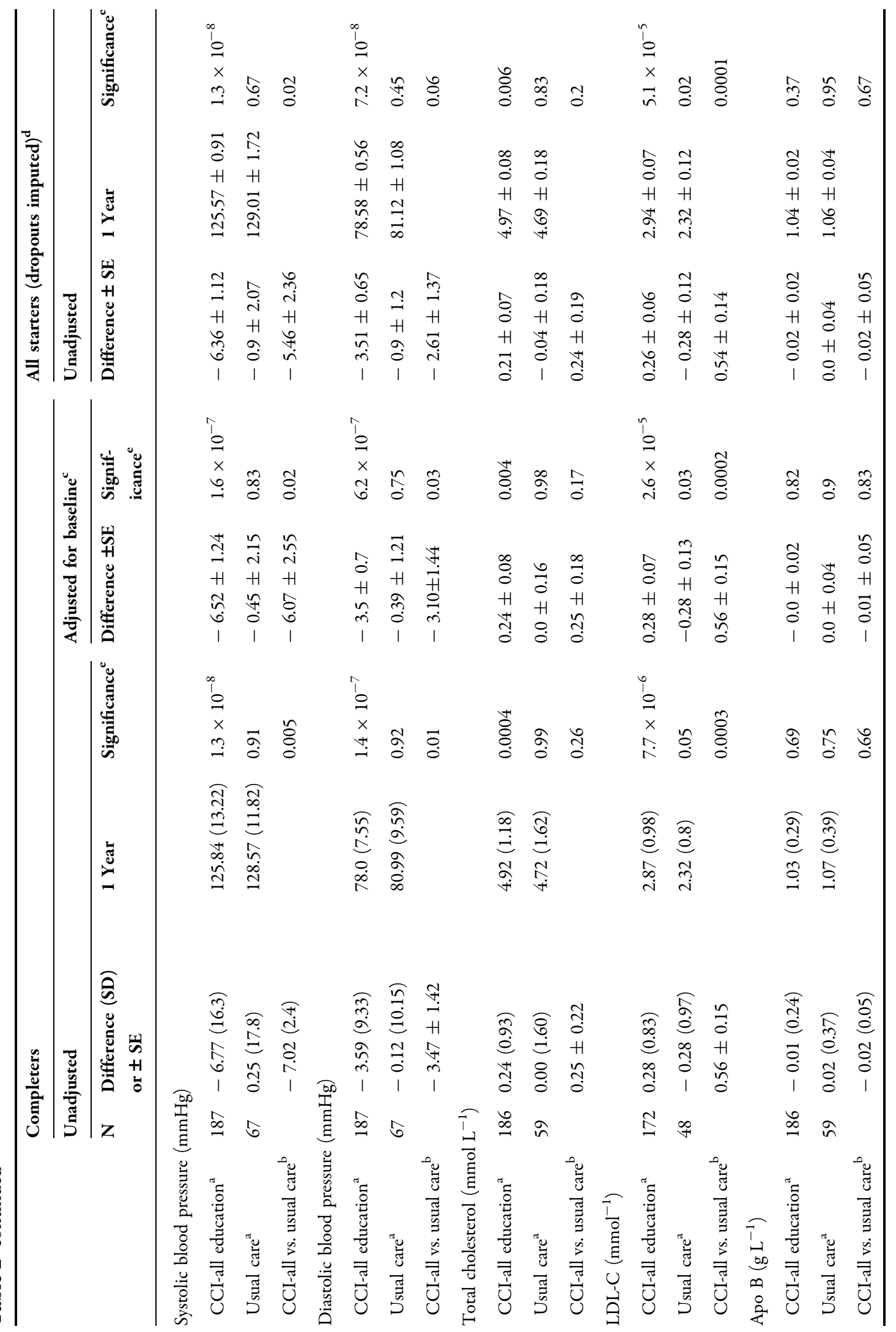




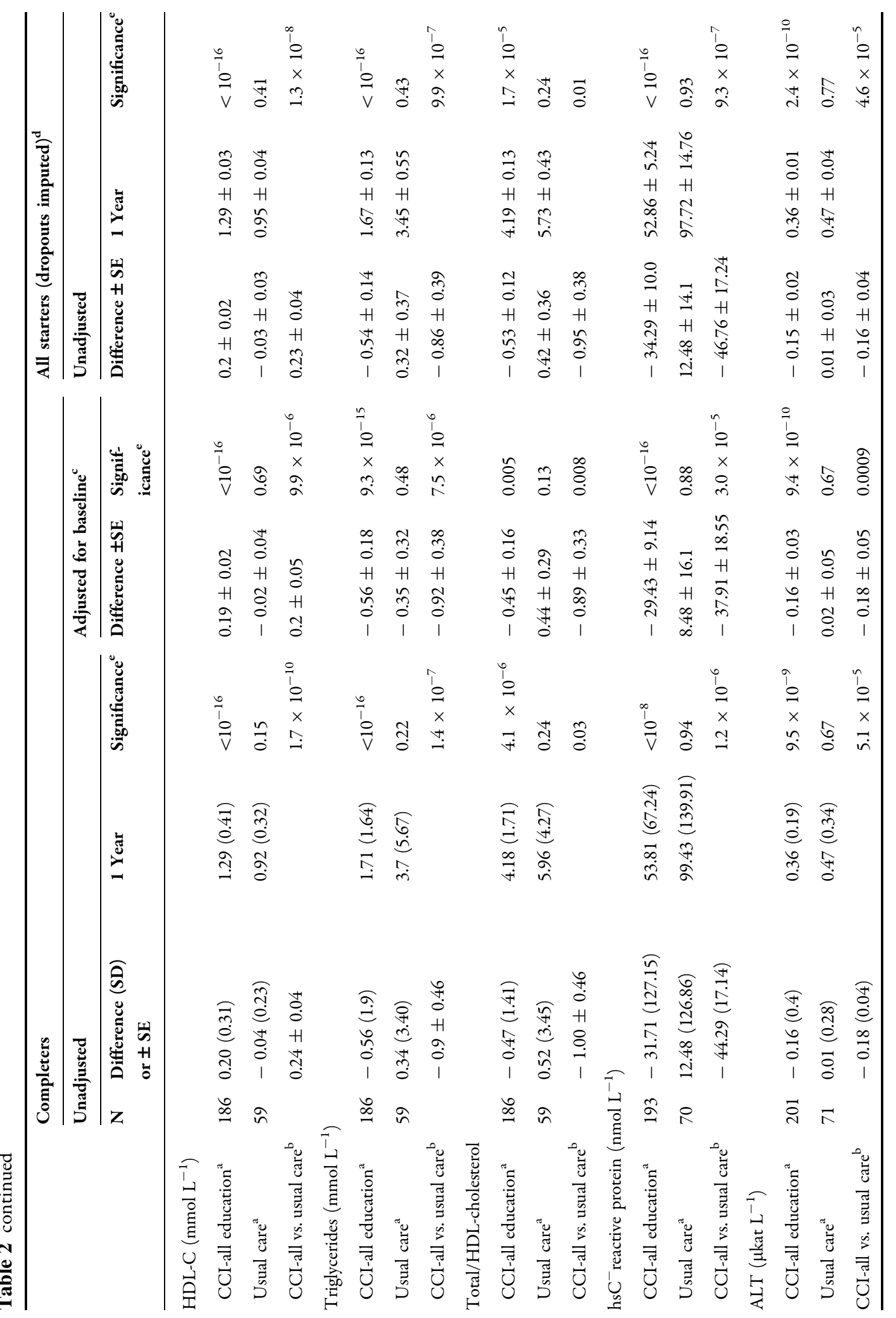




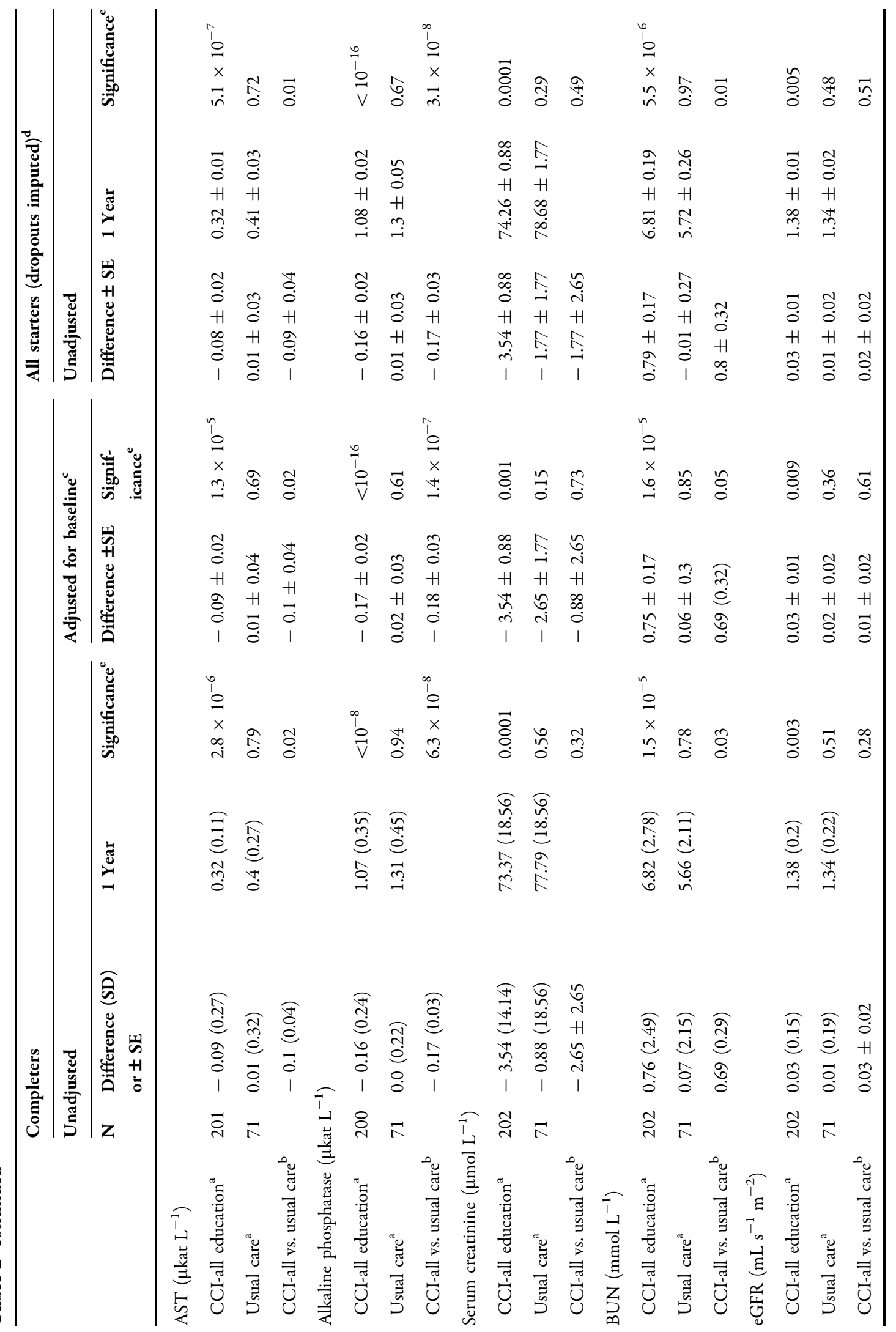




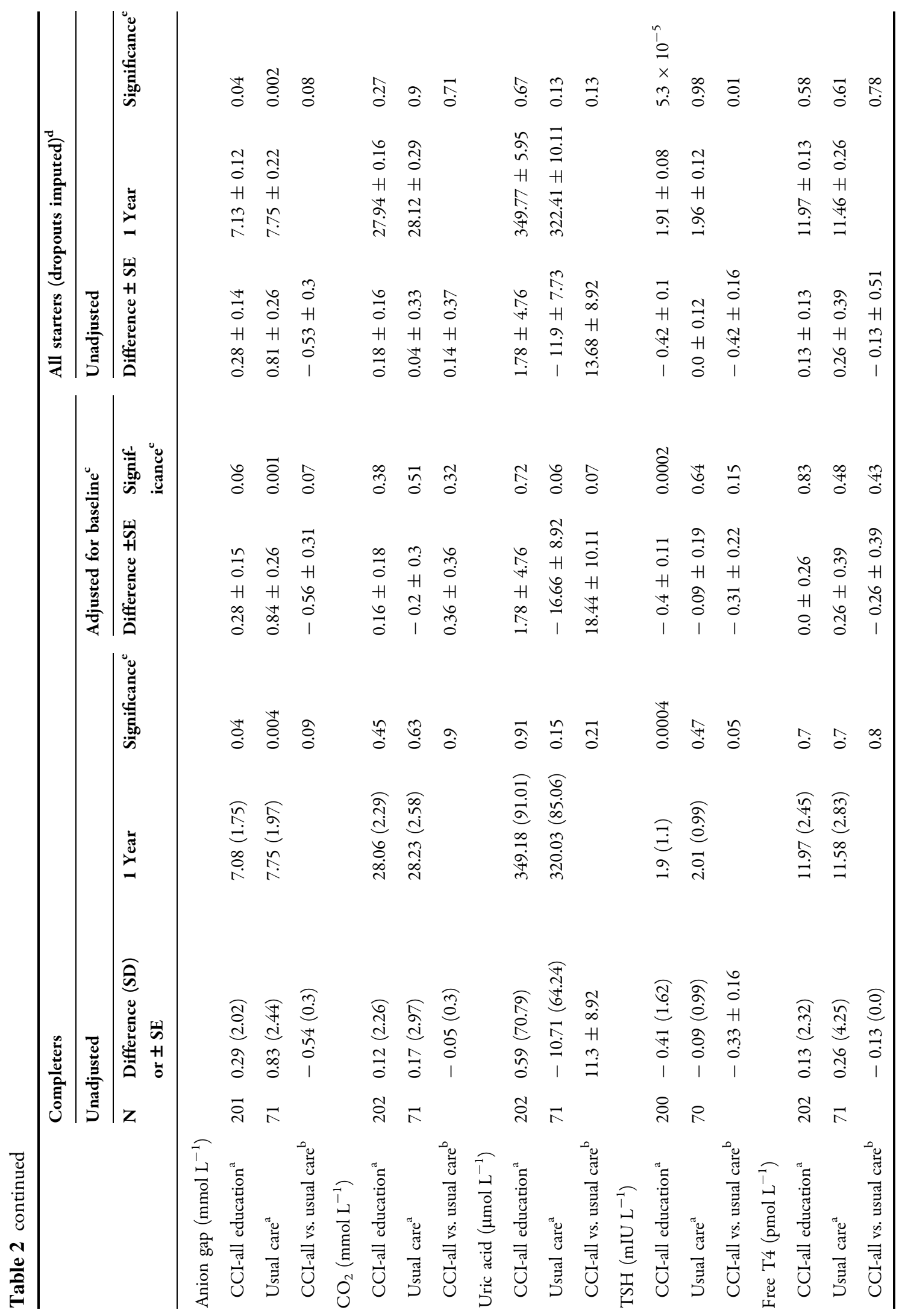




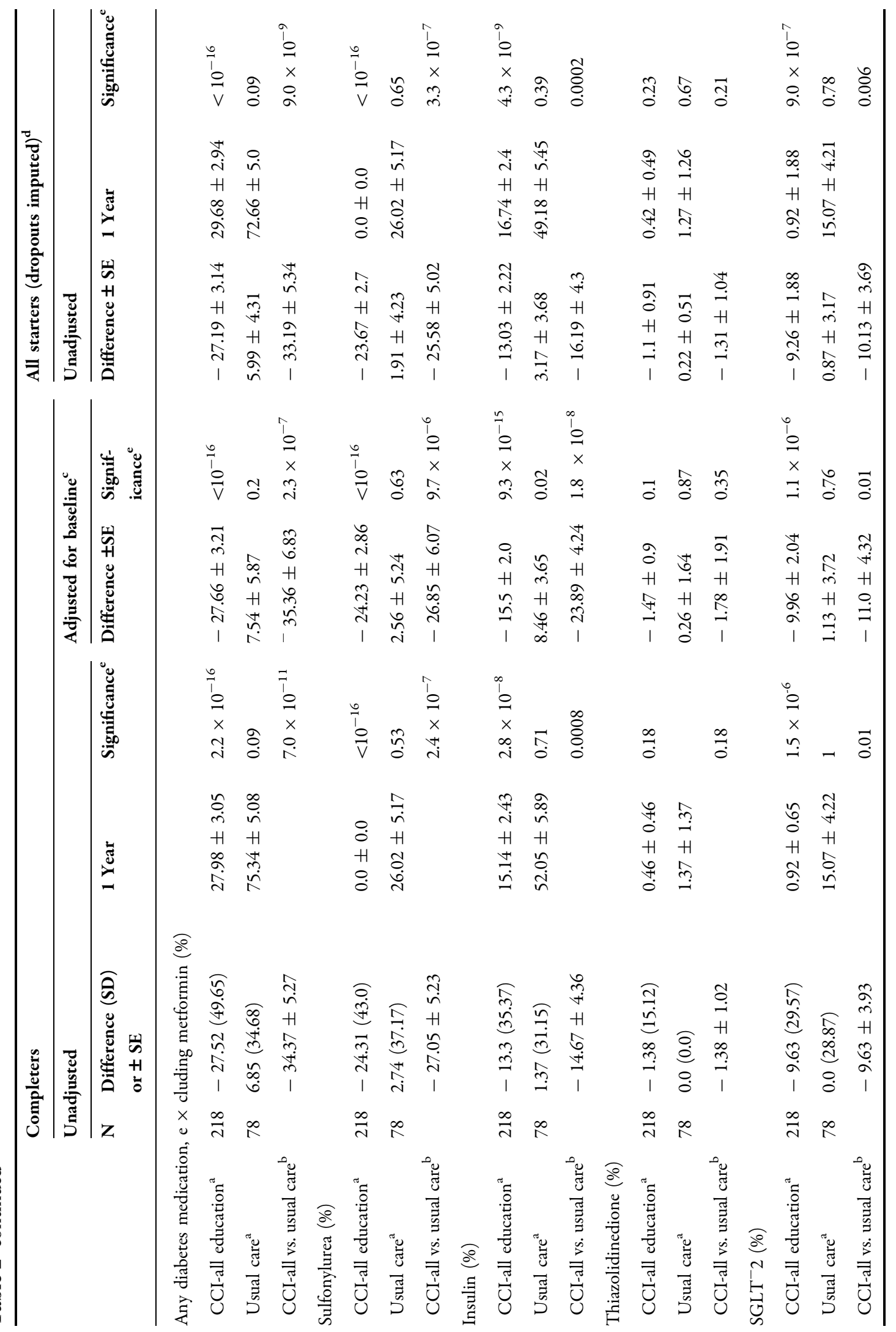




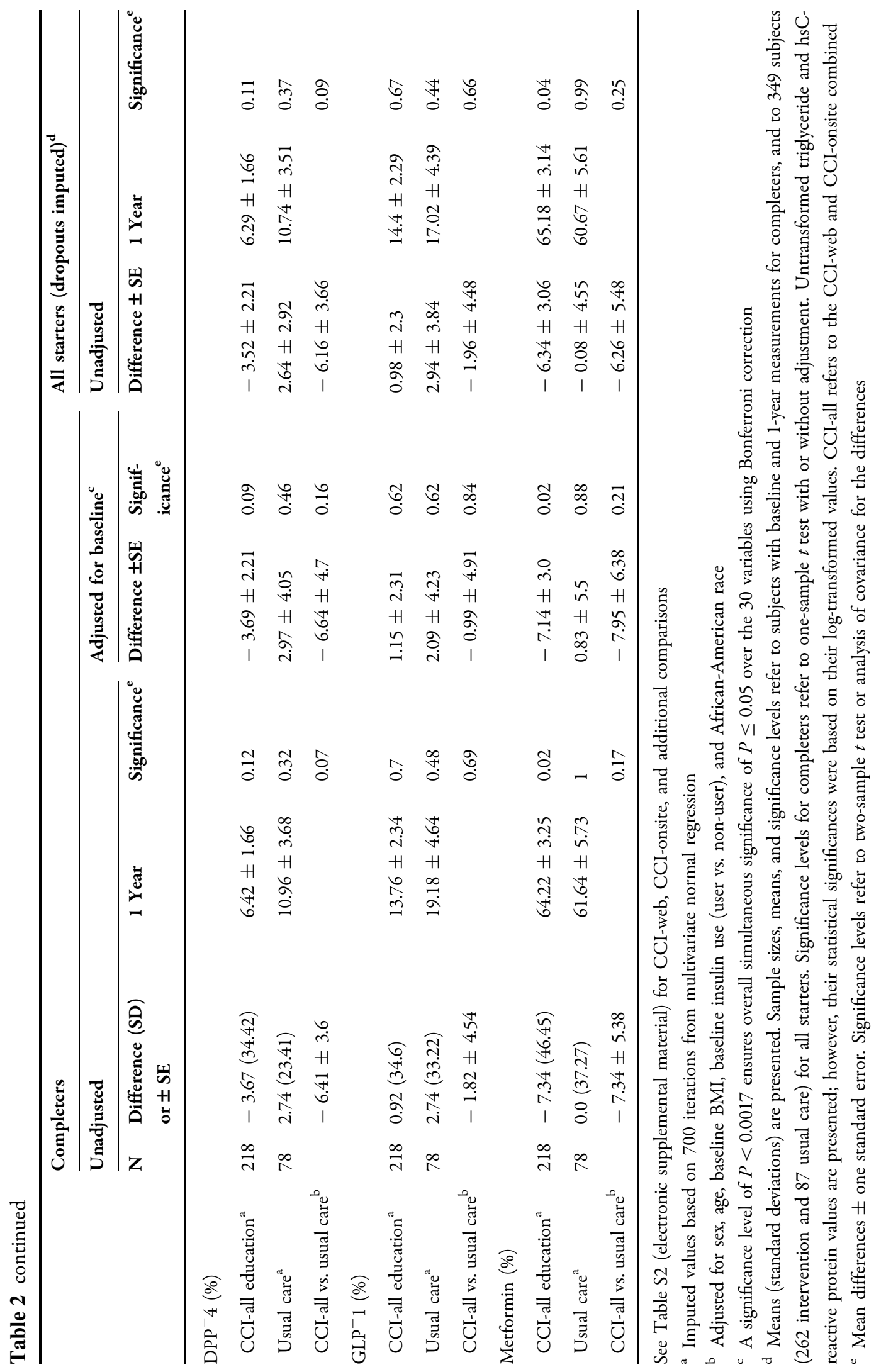


diabetes medication (57\% were prescribed a diabetes medication other than metformin, $30 \%$ prescribed insulin) and 93\% were obese. Eighty-seven percent of participants in UC at baseline were prescribed diabetes medication (46\% prescribed insulin), and 82\% were obese. Forty-four participants (16.8\%) withdrew from the CCI, 22 from each education delivery mode. Baseline characteristics of CCI dropouts did not differ significantly from the 218 completers except none of the five thiazolidinedione users were dropouts (Table 1). At baseline, characteristics of CCI participants who self-selected webbased versus on-site education were not significantly different after accounting for multiple comparisons (see Table S1 in the electronic supplementary material). Compared to the 78 UC participants who completed the study, the nine that withdrew tended to be older ( 58 versus 52 years old), had lower TSH, and fewer were prescribed insulin, SGLT-2, DPP-4, GLP-1, or blood pressure medications (Table 1).

\section{Effectiveness}

Table 2 presents mean 1-year changes in biomarkers. In the $\mathrm{CCI}, \mathrm{HbA}_{1 \mathrm{c}}$ was significantly reduced $17 \%$, from $60 \pm 1.0 \mathrm{mmol} \mathrm{mol}^{-1}$ $(7.6 \pm 0.09 \%)$ at baseline to $45 \pm 0.8 \mathrm{mmol}$ $\mathrm{mol}^{-1}(6.3 \pm 0.07 \%)$ after 1 year (nominal significance $P<1.0 \times 10^{-16} ;$ Fig. 1$)$. Eighty-five percent $(174 / 204)$ of CCI participants completing 1-year $\mathrm{HbA}_{1 \mathrm{c}}$ testing observed a decline greater than $2.2 \mathrm{mmol} \mathrm{mol}^{-1}(>0.2 \%)$ in the measure. When adjusted for multiple comparisons, significant within-CCI reductions were observed in fasting glucose $(-22 \%, P<1.0 \times$ $\left.10^{-16}\right)$, fasting insulin $\left(-43 \%, P=6.7 \times 10^{-16}\right)$, C-peptide $\left(-23 \%, P=2.2 \times 10^{-16}\right)$, HOMA-IR derived from fasting insulin excluding exogenous users $\left(-55 \%, P=3.2 \times 10^{-5}\right)$, HOMA-IR derived from C-peptide $(-29 \%, \quad P=1.0 \times$ $\left.10^{-13}\right)$, weight from clinic measurements $\left(-12 \%, \quad P<1.0 \times 10^{-16}\right)$, weight from home scales ( $-13 \%, P<1.0 \times 10^{-16}$, Fig. 2$)$, triglycerides $\left(-24 \%, P<1.0 \times 10^{-16}\right)$, high-sensitivity C-reactive protein $\left(-39 \%, P<1.0 \times 10^{-16}\right)$, ALT $\left(-30 \%, \quad P=2.4 \times 10^{-10}\right)$, AST $(-21 \%$, $\left.P=5.1 \times 10^{-7}\right)$, and alkaline phosphatase
(-13\%, $\left.\quad P<1.0 \times 10^{-16}\right) . \quad$ HDL-cholesterol increased $18 \%\left(P<1.0 \times 10^{-16}\right)$ and calculated LDL-cholesterol increased 10\% $\left(P=5.1 \times 10^{-5}\right)$ while apolipoprotein $\mathrm{B}(\mathrm{ApoB})$ concentration was unchanged $(P=0.37)$ for participants in the CCI. There were no significant differences in mean biomarker changes between CCI-web and CCI-onsite (see Table S2 in the electronic supplementary material). In contrast to the CCI, patients enrolled in UC for 1 year showed no Bonferroni-adjusted significant change for any of the biomarkers measured (Table 2).

Following 1 year of CCI, usage of all diabetes medications combined (excluding metformin) was reduced significantly $(56.9 \pm 3.1 \%$ to 29.7 $\left.\pm 3.0 \%, \quad P<1.0 \times 10^{-16}\right)$ through decreased prescriptions for DPP-4 (9.9-6.3\%, $P=0.11)$, insulin $\left(29.8-16.7 \%, P=4.3 \times 10^{-9}\right)$, SGLT-2 inhibitors $\left(10.3-0.9 \%, P=9 \times 10^{-7}\right)$, sulfonylureas $\left(23.7-0 \%, P<1.0 \times 10^{-16}\right)$, and thiazolidinediones (1.5-0.4\%, $P=0.23$ ) (Fig. 3). GLP-1 prescriptions were statistically unchanged (13.4\% at baseline to $14.4 \%$ at 1 year, $P=0.67$ ), and metformin decreased slightly (71.4-65.0\%, $P=0.04)$ for CCI participants. Forty percent (31/78) of CCI participants who began the study with insulin prescriptions (average dose of 64.2 units) eliminated the medication, while the remaining $60 \%(47 / 78)$ of insulin users reduced daily dosage from 105.2 to 53.8 units $(P<0.0001)$. Patients enrolled in UC for 1 year showed no Bonferroni-adjusted significant change for prescription of medication. For the 34 UC participants that continued using insulin, the average daily dose increased from 96.0 to 111.9 units.

The proportion of participants in the total imputed CCI group with $\mathrm{HbA}_{1 \mathrm{c}}$ below $48 \mathrm{mmol}$ $\mathrm{mol}^{-1}(<6.5 \%)$ increased from $19.5 \pm 2.4 \%$ to $69.8 \pm 3.1 \%$. Of those in the CCI with $\mathrm{HbA}_{1 \mathrm{c}}$ reported at 1 year, $72 \%(147 / 204)$ achieved $\mathrm{HbA}_{1 \mathrm{c}}$ below $48 \mathrm{mmol} \mathrm{mol}^{-1}$ (6.5\%) and $60.3 \%$ (123/204) of participants achieved $\mathrm{HbA}_{1 \mathrm{c}}$ below $48 \mathrm{mmol} \mathrm{mol}^{-1}(<6.5 \%)$ while taking no diabetes medication or only metformin. Of those in the CCI with $\mathrm{HbA}_{1 \mathrm{c}}$ below $48 \mathrm{mmol} \mathrm{mol}^{-1}$ $(<6.5 \%)$ at 1 year, $42.3 \%(52 / 123)$ were prescribed no diabetes medication and $57.7 \%$ (71/ 123) were prescribed metformin only. The proportion of the total imputed CCI group with 


\section{(a)} Continuous Care Intervention

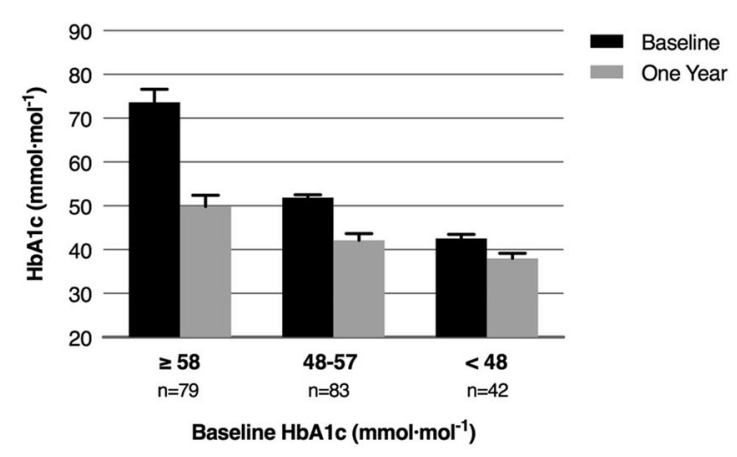

(b)

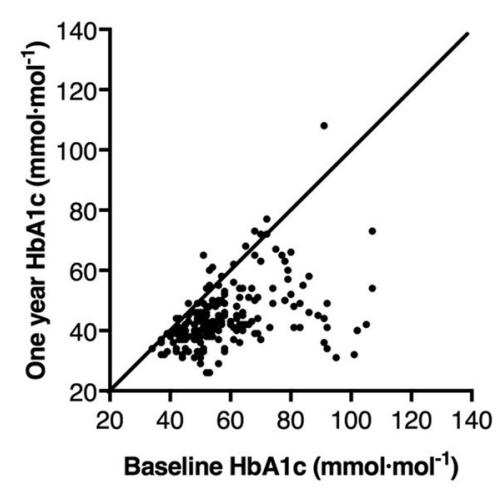

Fig. 1 Change in $\mathrm{HbA}_{1 \mathrm{c}}$ over the course of 1 year for CCI and UC groups. a Mean ( $95 \% \mathrm{CI}$ ) in $\mathrm{HbA}_{1 \mathrm{c}}$ based on starting value at baseline and 1 year for completers in both

fasting glucose below $6.99 \mathrm{mmol} \mathrm{L}^{-1}$ at 1 year increased from $34.9 \pm 3.3 \%$ to $58.4 \pm 3.9 \%$, and the proportion with class III obesity decreased from $45.5 \pm 3.1 \%$ to $19.6 \pm 2.8 \%$.

Compared to UC, the CCI showed significant Bonferroni-adjusted $(P<0.0017)$ net reductions in $\mathrm{HbA}_{1 \mathrm{c}}$ (nominal significance for the twogroup comparison, $P<10^{-16}$; Fig. 1), fasting glucose $\left(P=2.1 \times 10^{-6}\right)$, fasting insulin excluding exogenous users $\left(P=4.6 \times 10^{-5}\right)$, C-peptide $\left(P=5.3 \times 10^{-5}\right)$, HOMA-IR derived from insulin excluding exogenous users $\left(P=6.0 \times 10^{-5}\right)$ or derived from C-peptide $\left(P=3.0 \times 10^{-5}\right)$, weight $\left(P<10^{-16}\right)$, triglycerides $\left(P=1.0 \times 10^{-6}\right)$, hsCRP $\left(P=9.3 \times 10^{-7}\right)$, ALT $\left(P=4.6 \times 10^{-5}\right)$, and alkaline phosphatase $\left(P=3.1 \times 10^{-8}\right)$. All of these group differences remained significant when adjusted for the baseline age, sex, insulin medication use, and
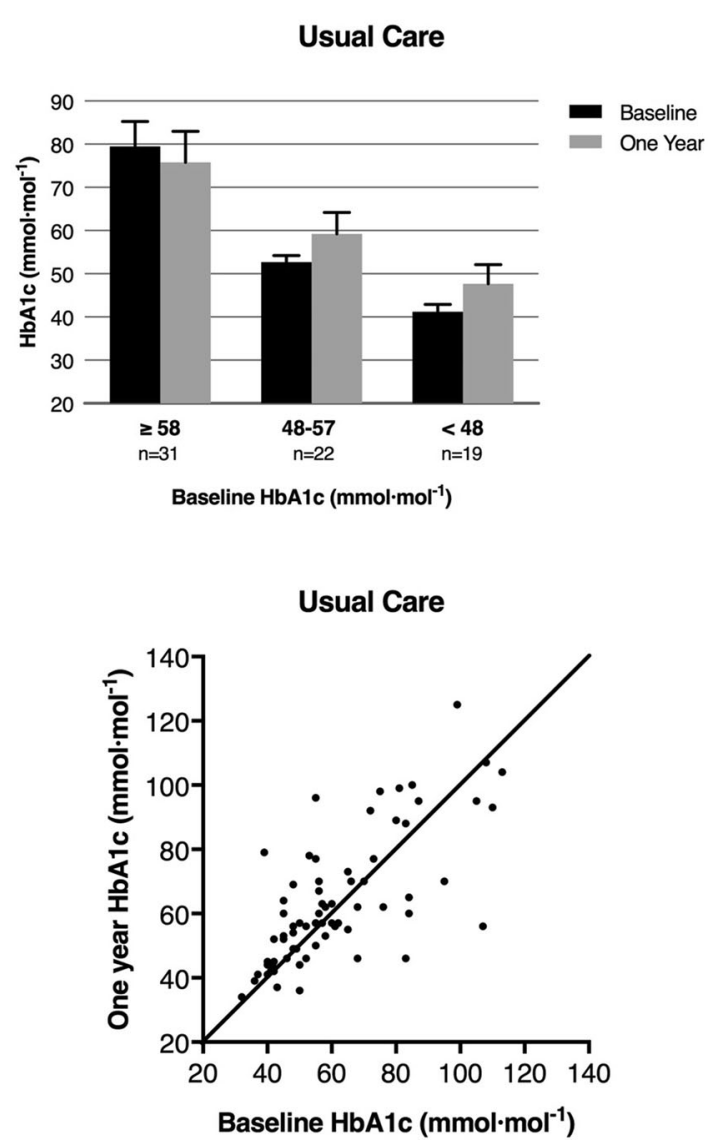

groups. b Individual changes in $\mathrm{HbA}_{1 \mathrm{c}}$ over 1 year for completers in both groups

body mass index (Table 2). The CCI decrease in diabetes medication use was significantly greater than the changes in the UC group for all diabetes medications $\left(P<10^{-16}\right)$ and all diabetes medications excluding metformin $\left(P=9.0 \times 10^{-9}\right), \quad$ including sulfonylurea $\left(P=3.3 \times 10^{-7}\right)$ and insulin $\quad(P=0.0002)$ (Fig. 3).

The CCI-web and CC-onsite sub-cohorts provide replication of the above results. Specifically, Table S2 (see electronic supplementary material) shows that within-group Bonferroni significance was achieved separately for the mean 1-year reductions in $\mathrm{HbA}_{1 \mathrm{c}}$, fasting glucose, fasting insulin, C-peptide, HOMA-IR, triglycerides, and hsCRP, and the significant increases in HDL-cholesterol and LDL-cholesterol. The Bonferroni-adjusted significant differences from the UC cohort were also 


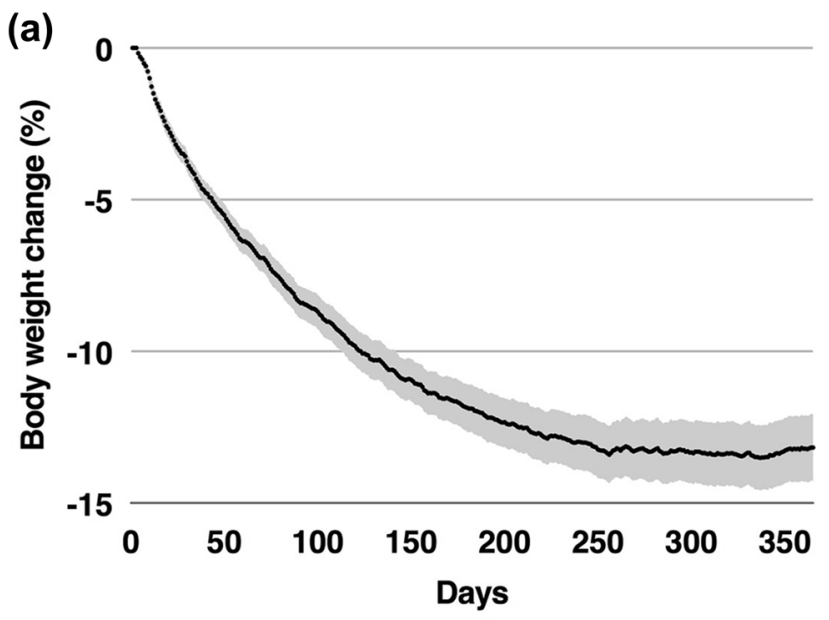

(b)

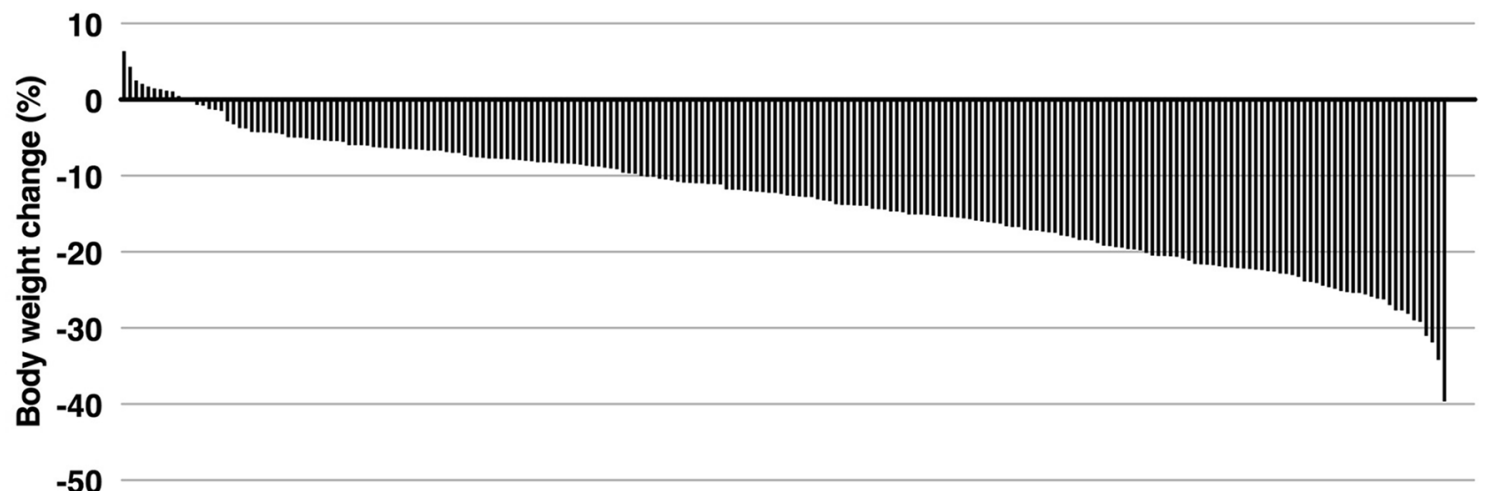

Fig. 2 Body weight change over the course of 1 year in CCI completers. a Mean ( $95 \%$ CI) change in body weight for completers over the course of 1 year. For each individual, weight on a given day was computed as the 3-day trailing mean (to reduce day-to-day variation). On

replicated by the two educational sub-cohorts for $\mathrm{HbA}_{1 \mathrm{c}}$, fasting glucose, insulin-derived HOMA-IR, weight, HDL-cholesterol, LDL-cholesterol, triglycerides, hsCRP, and alkaline phosphatase, with or without adjustment for baseline covariates.

\section{Time Course of Biomarker Change in CCI}

Over the course of the intervention at baseline, 70 days [23], and 1 year, the proportion of participants in the total imputed CCI with $\mathrm{HbA}_{1 \mathrm{c}}$ below $48 \mathrm{mmol} \mathrm{mol}^{-1}(<6.5 \%)$ increased from $19.5 \pm 2.4$ to $60.7 \pm 3.1$ to $69.8 \pm 3.1 \%$; the proportion with fasting glucose below dates where no weights were recorded during the 3-day time window for a given participant, the most recent 3-day mean preceding the date was used. $\mathbf{b}$ Histogram depicting individual body weight changes at 1 year

$6.99 \mathrm{mmol} \mathrm{L}^{-1}\left(<126 \mathrm{mg} \mathrm{dL}^{-1}\right)$ increased from $34.9 \pm 3.3$ to $55.5 \pm 3.3$ to $58.4 \pm 3.9 \%$, and the proportion with class III obesity decreased from $45.5 \pm 3.1$, to $30.2 \pm 3.1$, to $19.6 \pm 2.8 \%$.

The time course of biomarker changes also differed by variable (see Table S3 in the electronic supplementary material). Most of the 1-year improvements in diabetes risk factors were achieved during the first 70 days of the intervention including $84 \%$ of the $\mathrm{HbA}_{1 \mathrm{c}}$ decrease, $90 \%$ of the fasting glucose decrease, $73 \%$ of the fasting insulin decrease, $64 \%$ of the C-peptide decrease, and $87 \%$ and $74 \%$ of the decreases in HOMA-IR as estimated from fasting insulin and C-peptide concentrations, respectively. Improvements in blood pressure also mostly 
(a)

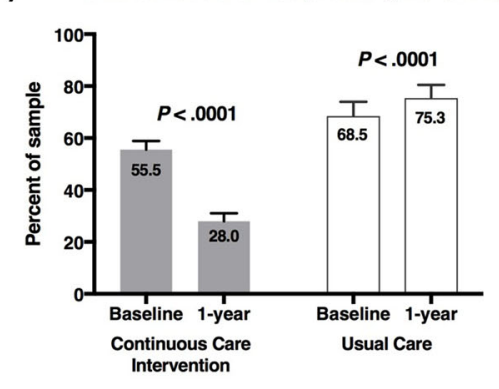

(b)

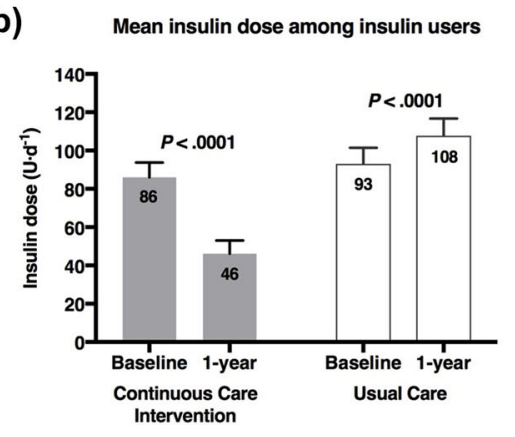

(c)
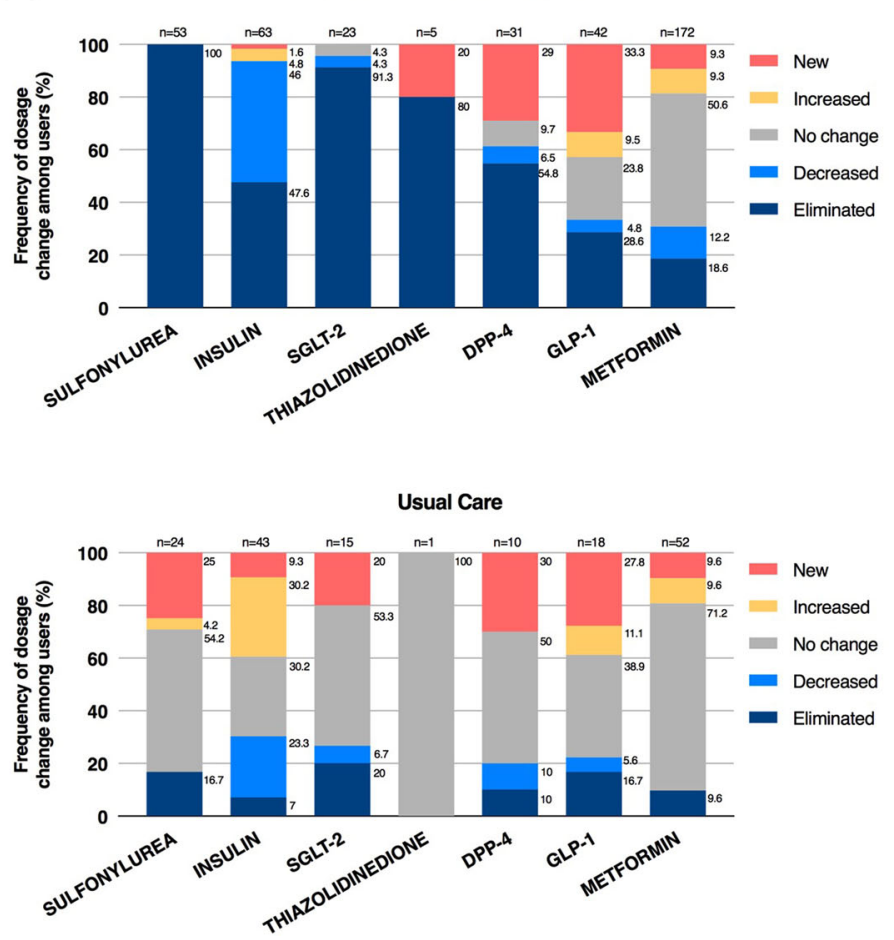

Fig. 3 Medication changes over the course of 1 year in completers of the CCI and UC groups. a Proportion of completers prescribed diabetes medications other than metformin. b Mean \pm SE prescribed dose among insulin

occurred in the initial 70 days, as did reductions in alkaline phosphatase, serum creatinine, and eGFR. Most of the plasma triglyceride decrease occurred during the first 70 days (87\%), whereas essentially all the substantial increase in HDL-cholesterol occurred between the initial 70 days of the intervention and 1 year (99\%). About $60 \%$ of weight loss occurred in the first 70 days.

\section{Retention and Adherence in CCI}

Eighty-three percent of participants remained enrolled in the CCI at 1 year. Nearly all CCI participants $(96 \%)$ reported at least one $\mathrm{BHB}$ reading of $0.5 \mathrm{mmol} \mathrm{L}^{-1}$ or more by handheld measure, and among completers, the group mean at 70 days by laboratory measure was over threefold the baseline $(0.54 \pm 0.04$ versus $\left.0.17 \pm 0.01 \mathrm{mmol} \mathrm{L}^{-1}\right)$. Laboratory-measured BHB at 1 year $\left(0.31 \pm 0.03 \mathrm{mmol} \mathrm{L}^{-1}\right)$ was nearly double the baseline value (Fig. 4). The users. $\mathbf{c}$ Frequency in change of medication dosage among prescribed users by diabetes medication class in both groups

intention-to-treat analysis yielded similar results, with an increase in average from baseline $\quad\left(0.17 \pm 0.01 \mathrm{mmol} \mathrm{L}^{-1}\right) \quad$ to 70 days $\left(0.54 \pm 0.04 \mathrm{mmol} \mathrm{L}^{-1}\right)$, followed by a decrease at 1 year $\left(0.30 \pm 0.02 \mathrm{mmol} \mathrm{L}^{-1}\right)$, though still nearly twofold the baseline concentrations.

\section{Safety and Adverse Events}

For CCI participants, acid-base physiology was normal; no cases of metabolic acidosis were observed. One CCI patient ( $0.38 \%$ of starters) had a clinically significant rise in serum creatinine, but group mean declined at 1 year. Mean blood urea nitrogen increased significantly in the CCI group, possibly indicating increased dietary protein consumption although high protein intake was not recommended. Mean uric acid in the CCI rose transiently at 70 days, but was unchanged at 1 year; no new cases of gout were diagnosed. Mean free T4 level was 


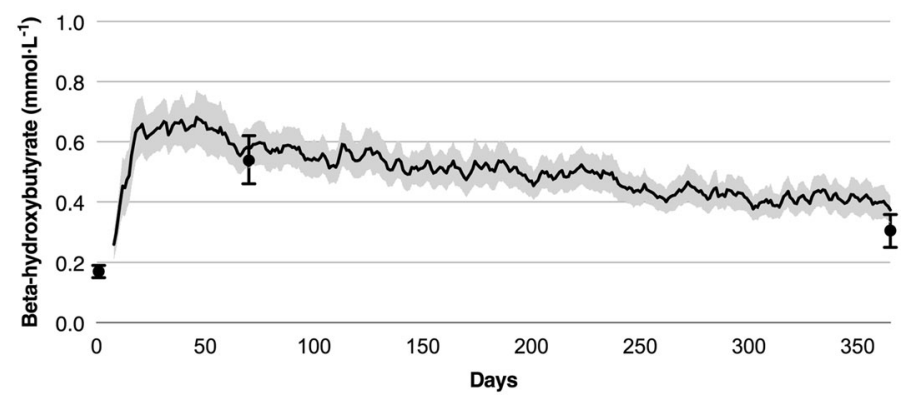

Fig. 4 Beta-hydroxybutyrate concentrations of CCI completers. Note: For each individual in the graph, the BHB concentration on a given day was computed as the 3-day trailing mean (to reduce day-to-day variation). On dates where no $\mathrm{BHB}$ concentrations were recorded during the 3-day time window for a given participant, the most recent

unchanged, and TSH was significantly lower at 1 year; two new cases of subclinical hypothyroidism were observed $(0.76 \%$ of starters $)$ in the CCI [24].

Adverse events occurred in 6/262 CCI participants including one non-ST-segment myocardial infarction, one inferior myocardial ischemia by electrocardiogram, one metastatic neuroendocrine carcinoma, one malignant cancer with multiple brain lesions and lung tumor, and death from renal hemorrhage and failure and hyperkalemia. Also, one episode of hypoglycemia occurred following a motor vehicle accident and medical records indicated the patient was not taking insulin as prescribed; no other episodes of symptomatic hypoglycemia requiring assistance were reported. None of the adverse events were attributed to the intervention.

Adverse events were reported in 6/87 UC participants, including one percutaneous coronary intervention (PCI) to left anterior descending stenosis, one PCI to right coronary artery, two carotid endarterectomies (one of which was successful), multifactorial encephalopathy, and diabetic ketoacidosis with pulmonary emboli.

\section{DISCUSSION}

This study evaluated the effectiveness and safety of an alternative treatment model for T2D 3-day mean preceding the date was used. Line graph depicts mean $(95 \% \mathrm{CI})$ over time for BHB measured at home and reported via the app. Dots and error bars represent the mean \pm SE from laboratory measured $\mathrm{BHB}$ at baseline, 70 days, and 1 year

that utilized continuous remote care to provide a high level of outpatient support combined with individualized nutrition enabling longterm maintenance of behavioral and metabolic change via nutritional ketosis. This trial prospectively observed adults with T2D undergoing treatment via this novel care model and a comparison group of adults with T2D undergoing usual care treatment. Following 1 year of CCI, participants achieved a $14 \mathrm{mmol} \mathrm{mol}^{-1}$ $(1.3 \pm 0.1 \%)$ decline in $\mathrm{HbA}_{1 \mathrm{c}}$ concurrent with $12 \%$ weight loss and reduction in medication use. Consistent conclusions were reached with intention-to-treat analysis and analysis of completers. A usual care group showed no change in diabetes status or related biomarkers over the year.

\section{Effectiveness}

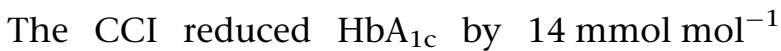
$(1.3 \%)$ at 1 year. $\mathrm{HbA}_{1 \mathrm{c}}$ reductions up to $7 \mathrm{mmol} \mathrm{mol}^{-1}(0.6 \%)$ via intensive lifestyle intervention [25] and $11 \mathrm{mmol} \mathrm{mol}^{-1}(1.0 \%)$ via an energy-restricted low-carbohydrate diet with partial food provision delivered via an outpatient setting [26] were previously reported. The present intervention achieved 12\% weight loss at 1 year; previously studied interventions elicited $4-9 \%$ weight loss in patients with T2D $[25,26]$. The regular monitoring of weight, glucose, and BHB as biometric feedback 
for participant, health coach, and medical provider may have provided behavior reinforcement. Further, it seems plausible that this multicomponent care model allowed for greater improvements compared to interventions that provided a subset of components. A recent primary care-led weight management intervention utilizing a 3-5 month VLCD resulted in a $10 \mathrm{mmol} \mathrm{mol}^{-1}(0.9 \%)$ reduction in $\mathrm{HbA}_{1 \mathrm{c}}$ and $10 \%$ weight loss at 1 year; $46 \%$ of participants achieved $\mathrm{HbA}_{1 \mathrm{c}}$ below $48 \mathrm{mmol} \mathrm{mol}{ }^{-1}(<6.5 \%)$ while taking no medications [27]. While only $25 \%$ of participants in the present investigation achieved this measure of diabetes remission, the protocol for the present investigation discontinued metformin prescription only because of contraindication, intolerance, or patient request given its efficacy for T2D prevention and recommended use in certain populations [7]. An additional $35 \%$ of participants in the present investigation were able to attain $\mathrm{HbA}_{1 \mathrm{c}}$ below $48 \mathrm{mmol} \mathrm{mol}^{-1}(<6.5 \%)$ while taking only metformin. The longer duration of T2D and baseline insulin prescription to $30 \%$ of participants might be factors influencing the proportion of participants in which glycemic control medications could be discontinued in this investigation.

$\mathrm{HbA}_{1 \mathrm{c}}$ improved concurrent with medication reductions prescribed for blood glucose-lowering. For each medication class, the sum percentage of eliminations and reductions of prescriptions at 1 year exceeded that observed at 70 days [23]. Improved glycemic control via a predominantly pharmaceutical approach has demonstrated paradoxical increased cardiovascular risk [28]. Tight glycemic control can elicit symptomatic hypoglycemia [29] or weight gain [30], neither of which was observed in CCI. Thus, it is likely the treatment method by which glycemic control is achieved (e.g., pharmacological, surgery, lifestyle intervention) is important to health outcomes and risk.

Most changes in $\mathrm{HbA}_{1 \mathrm{c}}$, glucose, insulin, C-peptide, and HOMA-IR occurred in the first 70 days with further improvement observed at 1 year. While the mechanism for improved insulin sensitivity in ketosis is not fully understood, early improvements in $\mathrm{HbA}_{1 \mathrm{c}}$ and HOMA-IR indicate rapid restoration of liver and peripheral insulin sensitivity and are consistent with improvements observed within 2 weeks of ketosis when measured by euglycemic hyperinsulinemic clamp [13]. Utilization of blood BHB for self-monitoring with reinforcement by clinicians may have contributed to sustained $\mathrm{HbA}_{1 \mathrm{c}}$ improvement. Further, BHB acts as a signaling molecule, reducing inflammation and oxidative stress [14, 15]; therefore, mild ketonemia may benefit multiple organs and systems. With appropriate dietary formulation, benefits of nutritional ketosis are observed in mouse models of longevity and health span [31, 32]. Participant mean BHB levels are of similar magnitude to those observed with SGLT-2 inhibitor treatment $\left(\sim 0.5 \mathrm{mmol} \mathrm{L}^{-1}\right) \quad$ [33]. Recent trials [5, 34] demonstrate cardiovascular benefits to two SGLT-2 inhibitors; mild ketosis was postulated as a mechanism [33]. Nutritionally achieved ketosis may have long-term cardiovascular benefits without the pharmaceutical risk profile [34]. Further, presence of glucose and palmitate has been associated with beta cell apoptosis [35]. Given the reduced levels of glucose and palmitate observed during nutritional ketosis [36], it is plausible that ketosis might play a role in attenuating glucolipotoxicity-induced beta cell death.

Beyond achieving improved glycemic control concurrent with medication and weight reductions, the CCI had broad positive impact on blood pressure, liver enzymes, hsCRP, triglycerides, and HDL-C. Elevated ALT, AST, and ALP are associated with non-alcoholic fatty liver disease and non-alcoholic steatohepatitis [37]; these enzymes were significantly reduced with intervention. Rapid reduction in triglycerides and gradual rise in HDL-C observed following CCI are consistent with previously studied carbohydrate-restricted interventions and carbohydrates are well known to increase triglycerides [38]. Of the 108 CCI completers with elevated baseline triglycerides $\left(\geq 1.69 \mathrm{mmol} \mathrm{L}^{-1}\right), 54 \%$ were in normal range at 1 year. Rise in LDL-C at 1 year, occurring with significant triglyceride decrease, was expected as there is less exchange via cholesteryl ester transfer protein [39]. However, this exchange would not affect particle number and ApoB was unchanged, suggesting an overall neutral 
impact on LDL lipoprotein-associated cardiovascular risk. In epidemiological studies, utilization of dietary saturated fat in place of carbohydrate was associated with beneficial impact on lipid profile, cardiovascular outcomes, and mortality despite higher LDL-C $[40,41]$. Transiently increased total and LDL cholesterol were also associated with mobilization of adipose cholesterol stores during major weight loss [42].

Consistent with population-level studies that observed very low rates of diabetes remission [43], the UC group had no change in $\mathrm{HbA}_{1 \mathrm{c}}$ and other indicators of glycemic status and insulin resistance but a net increase in diabetes medication use. Laboratory tests were generally unremarkable with biomarkers not changing significantly. The same facilities and methodologies were used for both the CCI and UC participants indicating that the changes observed in CCI participants not observed in the UC participants are unlikely to be due to methodological changes in clinical or laboratory data capture.

Despite independent recruitment of the CCI and UC groups, most of their baseline characteristics including $\mathrm{HbA}_{1 \mathrm{c}}$ and years since diabetes diagnosis were not significantly different. To enable a comparison between the CCI and UC groups, covariate adjustment was utilized to adjust for differences in baseline characteristics including sex, age, baseline BMI, baseline insulin use (user vs. non-user), and African-American race. With or without baseline adjustment, the change over 1 year elicited in the CC and UC groups differ in all primary outcomes$\mathrm{HbA}_{1 \mathrm{c}}$, medication use, and weight-and most secondary outcomes including lipid profile, inflammation, and liver function. In general, the favorable changes observed in the CCI were not observed in the UC cohort. For example, of patients who obtained $\mathrm{HbA}_{1 \mathrm{c}}$ measurements at 1 year, $60 \%$ of CCI participants achieved a $\mathrm{HbA}_{1 \mathrm{c}}$ below $48 \mathrm{mmol} \mathrm{mol}^{-1}(<6.5 \%)$ while taking no diabetes medications or metformin only, whereas only $10 \%$ of UC participants achieved this status.

One interpretation of these results is that the differences in observed outcomes over the year are due to advantages of the CCI over usual care. This suggests a need to incorporate carbohydrate restriction and comprehensive, continuous remote care as options in current guidelines for patients with diabetes as evidence accumulates [44]. However, alternative explanations are possible that may account for the large degree of difference observed. For instance, patients entering the CCI were recruited knowing that they were making a commitment to lifestyle change, while the UC participants were identified as recent referrals to local diabetes education programs and may not have had similar motivation or expectations of effort as the CCI participants. However, even when motivation is controlled for upon recruitment as an inclusion criterion for participation, additional factors may play a role in retention as evidenced by a recent study with randomization [45]. Also, the CCI and UC cohorts may also have differed in baseline characteristics that were not captured such as socioeconomic status.

Additionally, the treatment intensity of the two cohorts was not equal. The UC participants had one or more meetings with a registered dietitian and were under the medical supervision of their primary care provider or endocrinologist with periodic medical visits. In contrast, the CCI participants received a comprehensive and individualized continuous remote care intervention (and in one subgroup, the addition of on-site group classes). A more intensive intervention might have delivered somewhat better results than the investigation's UC group. For instance, a recent in-person group-based intervention for weight loss in T2D adults reduced $\mathrm{HbA}_{1 \mathrm{c}}$ by $3 \mathrm{mmol} \mathrm{mol}{ }^{-1}(0.3 \%)$ and weight by $4.0 \%$ after a year and medications were reduced in $26 \%$ of participants [46]. Future research might compare interventions of similar intensity with different treatment strategies to begin to understand the contribution of each component of the intervention to the overall effect.

\section{Adherence to CCI}

Eighty-three percent of CCI participants were retained through 1 year; patient perceived 
benefits of favorable health outcomes, individualized continuity of care, relationship with health coach, ongoing education, biometric feedback, and peer support may have aided retention. Most participants achieved nutritional ketosis during CCI and maintained elevated $\mathrm{BHB}$ at 1 year, indicating sustainability and was possibly enabled by the novel use of blood BHB as daily biofeedback for adherence.

\section{Safety of CCI}

No episodes of ketoacidosis, no hypo- or hyperglycemic events requiring assistance, and no adverse events were attributable to the CCI. With improvements or no change in liver, kidney, and thyroid function, safety of the intervention appears favorable. The absence of hypoglycemic events requiring assistance despite relatively tight glucose control may be due to the careful medical provider prescription management, especially rapid downward titration of insulin and sulfonylurea preventing hypoglycemia following dietary changes. Additionally, elevated BHB may have offered protection against hypoglycemic events, as starvation-adapted humans with elevated BHB have demonstrated full preservation of central nervous system function despite profound hypoglycemia induced by exogenous insulin [47].

\section{Study Strengths and Weaknesses}

Prior studies have demonstrated favorable improvements in T2D status following carefully managed ketogenic diets as case series [48] or in small short-term randomized trials [45]. This study's strengths include its prospective design, large cohort, high retention, duration, replication of findings between the CCI-onsite and CCI-web groups, and the collection of multiple time points in the intervention group allowing assessment of how biomarkers changed over time. This study also included participants prescribed insulin and with long-standing T2D, which were often exclusion criteria for prior studies. The means of recruitment, outpatient setting, and lack of food provision may enhance the real-world application of this study.
Weaknesses of this study include that it occurred at a single site and participants were mostly Caucasian. Socioeconomic and psychosocial status and genetics data were not collected. The study was not of sufficient size and duration to measure hard endpoints (e.g., mortality). Future trials could include a multisite randomized controlled trial with greater racial and ethnic diversity, broader age range, and greater disease severity.

\section{CONCLUSIONS}

This study demonstrated that a T2D intervention combining technology-enabled continuous remote care with individualized care plans encouraging nutritional ketosis can significantly reduce $\mathrm{HbA}_{1 \mathrm{c}}$, medication use, and weight within 70 days [23], and that these outcomes can be maintained or improved through 1 year. Most intervention participants with $\mathrm{HbA}_{1 \mathrm{c}}$ reported at 1 year achieved glycemic control in the sub-diabetes range with either no medication or the use of metformin alone. Related health parameters improved including blood pressure, lipid-lipoprotein profile, inflammation, and liver function. Ongoing research will determine the continued sustainability, effectiveness, and safety of these behavioral and metabolic changes.

\section{ACKNOWLEDGEMENTS}

The authors thank the participants for their commitment to health and advancing scientific knowledge. Special thanks to IUH staff, especially Monica Keyes, Danielle Wharff, Patti McKee, Joni Anderson, and Zachary Roberts, for their excellent patient care, as well as Rachel Bolden, Sydney Rivera, and Deklin Veenhuizen, who supported study logistics. We appreciate the health coaches who worked one-on-one with participants to help them achieve their goals-Brent Creighton PhD, Theresa Link RD, Bobbie Glon RN, and Marcy Abner RD. We are grateful for Roxie McKee and her staff at Indiana University Health Arnett Laboratory Services and Dave Gibson and Jennifer L. Powers and 
their staff at Washington University CLCS for their roles in sample analysis, as well as Teryn Sapper and staff from the Volek Laboratory at Ohio State University for their assistance with sample storage and transportation logistics.

Funding. Sponsorship for this study and article processing charges were funded by Virta Health Corp., San Francisco, CA, USA.

Authorship. All named authors meet the International Committee of Medical Journal Editors (ICMJE) criteria for authorship for this article, take responsibility for the integrity of the work as a whole, and have given their approval for this version to be published. Contributions: conceptualization and methodology, Sarah J. Hallberg, Amy L. McKenzie, Wayne W. Campbell, Tamara Hazbun, Brittanie M. Volk, James P. McCarter, Stephen D. Phinney, and Jeff S. Volek; formal analysis, Paul Williams; investigation, Sarah J. Hallberg, Amy L. McKenzie, Tamara Hazbun, Brittanie M. Volk; Writing-Original Draft, Amy L. McKenzie, Paul Williams, James P. McCarter; writing-review and editing, Sarah J. Hallberg, Amy L. McKenzie, Paul Williams, Nasir H. Bhanpuri, Anne L. Peters, Wayne W. Campbell, Tamara Hazbun, Brittanie M. Volk, James P. McCarter, Stephen D. Phinney, Jeff S. Volek; visualization, Amy L. McKenzie, Paul Williams, Nasir H. Bhanpuri, Stephen D. Phinney. All authors had full access to all of the data in this study and take complete responsibility for the integrity of the data and accuracy of the data analysis.

Disclosures. Virta Health Corp. funded this research study. Sarah J. Hallberg is an employee of Virta Health Corp. and has been offered stock options. Amy L. McKenzie is an employee of Virta Health Corp. and has been offered stock options. Nasir H. Bhanpuri is an employee of Virta Health Corp. and has been offered stock options. Brittanie M. Volk is an employee of Virta Health Corp. and has been offered stock options. James P. McCarter is an employee of Virta Health Corp. and has been offered stock options. Stephen D. Phinney is an employee of Virta Health Corp. and has been offered stock options. Stephen D. Phinney is a founder of
Virta Health Corp. Jeff S. Volek is also a founder of Virta Health Corp. Paul Williams is a paid consultant of Virta Health Corp. Anne L. Peters has been an advisory board member or has consulted for Abbott Diabetes Care, Becton-Dickinson, Bigfoot, Boehringer Ingelheim, Dexcom, Eli Lilly and Company, Janssen, Lexicon, Medscape, Merck, Novo Nordisk, sanofi, and Science 37, and has been on the Speaker's Bureau for NovoNordisk in the last year. Wayne W. Campbell and Tamara Hazbun have nothing to disclose.

Compliance with Ethics Guidelines. All procedures performed in studies involving human participants were in accordance with the ethical standards of the institutional and/or national research committee and with the 1964 Helsinki declaration and its later amendments or comparable ethical standards. Informed consent was obtained from all individual participants included in the study.

Data Availability. The data sets analyzed during the current study are available from the corresponding author on reasonable request.

Open Access. This article is distributed under the terms of the Creative Commons Attribution-NonCommercial 4.0 International License (http://creativecommons.org/licenses/ by-nc/4.0/), which permits any noncommercial use, distribution, and reproduction in any medium, provided you give appropriate credit to the original author(s) and the source, provide a link to the Creative Commons license, and indicate if changes were made.

\section{REFERENCES}

1. NCD Risk Factor Collaboration (NCD-RisC). Worldwide trends in diabetes since 1980: a pooled analysis of 751 population-based studies with 4.4 million participants. Lancet. 2016;387(10027): 1513-30.

2. Stokes A, Preston SH. Deaths attributable to diabetes in the united states: comparison of data sources and estimation approaches. PLoS One. 2017;12(1):e0170219. 
3. Gregg EW, Chen H, Wagenknecht LE, et al. Association of an intensive lifestyle intervention with remission of type 2 diabetes. JAMA. 2012;308(23):2489-96.

4. Marso SP, Daniels GH, Brown-Frandsen K, et al. Liraglutide and cardiovascular outcomes in type 2 diabetes. N Engl J Med. 2016;375(4):311-22.

5. Zinman B, Wanner C, Lachin JM, et al. Empagliflozin, cardiovascular outcomes, and mortality in type 2 diabetes. $\mathrm{N}$ Engl J Med. $2015 ; 373(22): 2117-28$.

6. Schauer PR, Bhatt DL, Kirwan JP, et al. Bariatric surgery versus intensive medical therapy for diabetes-3-year outcomes. N Engl J Med. 2014;370(21):2002-13.

7. American Diabetes Association. Standards of medical care in diabetes-2018. Diabetes Care. 2018;41(Supplement 1):S1-172.

8. Handelsman Y, Henry RR, Bloomgarden ZT, et al. American Association of Clinical Endocrinologists and American College of Endocrinology position statement on the association of SGLT-2 inhibitors and diabetic ketoacidosis. Endocr Pract. 2016;22(6):753-62.

9. Mottalib A, Sakr M, Shehabeldin M, Hamdy O. Diabetes remission after nonsurgical intensive lifestyle intervention in obese patients with type 2 diabetes. J Diabetes Res. 2015;2015(2):1-4.

10. Tobias DK, Chen M, Manson JE, Ludwig DS, Willett W, Hu FB. Effect of low-fat diet interventions versus other diet interventions on long-term weight change in adults: a systematic review and metaanalysis. Lancet Diabetes Endocrinol. 2015;3(12):968-79.

11. Chaudhry ZW, Doshi RS, Mehta AK, et al. A systematic review of commercial weight loss programmes' effect on glycemic outcomes among overweight and obese adults with and without type 2 diabetes mellitus. Obes Rev. 2016;17(8):758-69.

12. Bistrian BR, Blackburn GL, Flatt JP, Sizer J, Scrimshaw NS, Sherman M. Nitrogen metabolism and insulin requirements in obese diabetic adults on a protein-sparing modified fast. Diabetes. 1976;25(6):494-504.

13. Boden G, Sargrad K, Homko C, Mozzoli M, Stein TP. Effect of a low-carbohydrate diet on appetite, blood glucose levels, and insulin resistance in obese patients with type 2 diabetes. Ann Intern Med. 2005;142(6):403-11.

14. Shimazu T, Hirschey MD, Newman J, et al. Suppression of oxidative stress by $\beta$-hydroxybutyrate, an endogenous histone deacetylase inhibitor. Science. 2013;339(6116):211-4.

15. Youm Y-H, Nguyen KY, Grant RW, et al. The ketone metabolite $\beta$-hydroxybutyrate blocks NLRP3 inflammasome-mediated inflammatory disease. Nat Med. 2015;21(3):263-9.

16. Yancy WS, Olsen MK, Guyton JR, Bakst RP, Westman EC. A low-carbohydrate, ketogenic diet versus a low-fat diet to treat obesity and hyperlipidemia: a randomized, controlled trial. Ann Intern Med. 2004;140(10):769-77.

17. Westman EC, Yancy WS, Mavropoulos JC, Marquart M, McDuffie JR. The effect of a low-carbohydrate, ketogenic diet versus a low-glycemic index diet on glycemic control in type 2 diabetes mellitus. Nutr Metab. 2008;5(1):36.

18. Nielsen JV, Joensson EA. Low-carbohydrate diet in type 2 diabetes: stable improvement of bodyweight and glycemic control during 44 months follow-up. Nutr Metab. 2008;5(1):14-6.

19. Saslow LR, Kim S, Daubenmier JJ, et al. A randomized pilot trial of a moderate carbohydrate diet compared to a very low carbohydrate diet in overweight or obese individuals with type 2 diabetes mellitus or prediabetes. PLoS One. 2014;9(4):1-11.

20. Bazzano LA, Hu T, Reynolds K, et al. Effects of lowcarbohydrate and low-fat diets. Ann Intern Med. 2014;161(5):309-18.

21. Wagner EH, Austin BT, Davis C, Hindmarsh M, Schaefer J, Bonomi A. Improving chronic illness care: translating evidence into action. Health Aff. 2001;20(6):64-78.

22. Institute of Medicine. Dietary reference intakes for energy, carbohydrate. Fiber, fat, fatty acids, cholesterol, protein, and amino acids. Washington: The National Academies Press; 2005. p. 1-1357.

23. McKenzie A, Hallberg S, Creighton BC, et al. A novel intervention including individualized nutritional recommendations reduces hemoglobin A1c level, medication use, and weight in type 2 diabetes. JMIR Diabetes. 2017;2(1):e5.

24. Chubb SAP, Davis WA, Inman Z, Davis TME. Prevalence and progression of subclinical hypothyroidism in women with type 2 diabetes: the Fremantle Diabetes Study. Clin Endocrinol (Oxf). 2005;62(4):480-6.

25. The Look AHEAD Research Group. Reduction in weight and cardiovascular disease risk factors in individuals with type 2 diabetes: one-year results of the look AHEAD trial. Diabetes Care. 2007;30(6):1374-83. 
26. Tay J, Luscombe-Marsh ND, Thompson $\mathrm{CH}$, et al. Comparison of low- and high-carbohydrate diets for type 2 diabetes management: a randomized trial. Am J Clin Nutr. 2015;102(4):780-90.

27. Lean ME, Leslie WS, Barnes AC, et al. Primary careled weight management for remission of type 2 diabetes (DiRECT): an open-label, cluster-randomised trial. Lancet. 2017. https://doi.org/10. 1016/S0140-6736(17)33102-1

28. The ACCORD Study Group. Long-term effects of intensive glucose lowering on cardiovascular outcomes. N Engl J Med. 2011;364(9):818-28.

29. Action to Control Cardiovascular Risk in Diabetes Study Group, Gerstein HC, Miller ME, Byington RP, Goff DC, Bigger JT. Effects of intensive glucose lowering in type 2 diabetes. $\mathrm{N}$ Engl J Med. 2008;358(24):2545-59.

30. Henry RR, Gumbiner B, Ditzler T, Wallace P, Lyon $\mathrm{R}$, Glauber HS. Intensive conventional insulin therapy for type II diabetes. Metabolic effects during a 6-mo outpatient trial. Diabetes Care. $1993 ; 16(1): 21-31$.

31. Roberts MN, Wallace MA, Tomilov AA, et al. A ketogenic diet extends longevity and healthspan in adult mice. Cell Metabolism. 2017;26(3):539-46.

32. Newman JC, Covarrubias AJ, Zhao M, et al. Ketogenic diet reduces midlife mortality and improves memory in aging mice. Cell Metab. 2017;26(3):547-8.

33. Ferrannini E, Mark M, Mayoux E. CV protection in the EMPA-REG outcome trial: a "thrifty substrate" hypothesis. Diabetes Care. 2016;39(7):1108-14.

34. Neal B, Perkovic V, Mahaffey KW, et al. Canagliflozin and cardiovascular and renal events in type 2 diabetes. N Engl J Med. 2017;377(7):644-57.

35. El-Assaad W, Buteau J, Peyot M-L, et al. Saturated fatty acids synergize with elevated glucose to cause pancreatic $\beta$-cell death. Endocrinology. 2003;144(9):4154-63.

36. Forsythe CE, Phinney SD, Feinman RD, et al. Limited effect of dietary saturated fat on plasma saturated fat in the context of a low carbohydrate diet. Lipids. 2010;45(10):947-62.

37. Younossi ZM, Gramlich T, Matteoni CA, Boparai N, McCullough AJ. Nonalcoholic fatty liver disease in patients with type 2 diabetes. Clin Gastroenterol Hepatol. 2004;2(3):262-5.
38. Gardner CD, Kiazand A, Alhassan S, et al. Comparison of the Atkins, Zone, Ornish, and LEARN diets for change in weight and related risk factors among overweight premenopausal women. JAMA. 2007;297(9):969-77.

39. Barter PJ, Brewer HB, Chapman MJ, Hennekens $\mathrm{CH}$, Rader DJ, Tall AR. Cholesteryl ester transfer protein: a novel target for raising HDL and inhibiting atherosclerosis. Arterioscler Thromb Vasc Biol. 2003;23(2):160-7.

40. Dehghan M, Mente A, Zhang X, et al. Associations of fats and carbohydrate intake with cardiovascular disease and mortality in 18 countries from five continents (PURE): a prospective cohort study. Lancet. 2017;390(10107):2050-62.

41. Mente A, Dehghan M, Rangarajan S, et al. Association of dietary nutrients with blood lipids and blood pressure in 18 countries: a cross-sectional analysis from the PURE study. Lancet Diabetes Endocrinol. 2017;5(10):774-87.

42. Phinney SD, Tang AB, Waggoner CR, Tezanos-Pinto RG, Davis PA. The transient hypercholesterolemia of major weight loss. Am J Clin Nutr. 1991;53(6):1404-10.

43. Karter AJ, Nundy S, Parker MM, Moffet HH, Huang ES. Incidence of remission in adults with type 2 diabetes: the diabetes \& aging study. Diabetes Care. 2014;37(12):3188-95.

44. Noakes TD, Windt J. Evidence that supports the prescription of low-carbohydrate high-fat diets: a narrative review. Br J Sports Med. 2017;51(2):133-9.

45. Saslow LR, Mason AE, Kim S, et al. An online intervention comparing a very low-carbohydrate ketogenic diet and lifestyle recommendations versus a plate method diet in overweight individuals with type 2 diabetes: a randomized controlled trial. J Med Internet Res. 2017;19(2):e36.

46. O’Neil PM, Miller Kovach K, Tuerk PW, et al. Randomized controlled trial of a nationally available weight control program tailored for adults with type 2 diabetes. Obesity. 2016;24(11):2269-77.

47. Drenick EJ, Alvarez LC, Tamasi GC, Brickman AS. Resistance to symptomatic insulin reactions after fasting. J Clin Invest. 1972;51(10):2757-62.

48. Dashti HM, Mathew TC, Khadada M, et al. Beneficial effects of ketogenic diet in obese diabetic subjects. Mol Cell Biochem. 2007;302(1-2):249-56. 